BRAFT VERSion January 27, 2021

Typeset using $\mathrm{LAT}_{\mathrm{E}} \mathrm{X}$ default style in AASTeX63

\title{
3HWC: The Third HAWC Catalog of Very-High-Energy Gamma-ray Sources
}

A. Albert (D) ${ }^{1}$ R. Alfaro (D), ${ }^{2}$ C. Alvarez,${ }^{3}$ J.D. Álvarez,${ }^{4}$ J.R. Angeles Camacho, ${ }^{2}$ J.C. Arteaga-Velázquez,${ }^{4}$ K.P. Arunbabu, ${ }^{5}$ D. Avila Rojas, ${ }^{2}$ H.A. Ayala Solares $\left(\mathbb{D},{ }^{6}\right.$ V. Baghmanyan $\mathbb{D},{ }^{7}$ E. Belmont-Moreno (D) ${ }^{2}$ S.Y. BenZvi $\mathbb{D}^{8},{ }^{8}$ C. Brisbois (iD, ${ }^{9}$ K.S. Caballero-Mora $\left(\mathbb{D},{ }^{3}\right.$ T. Capistrán $\mathbb{D}^{1},{ }^{10}$ A. Carramiñana $\left(\mathbb{D},{ }^{10}\right.$ S. Casanova (iD, ${ }^{7}$ U. Cotti $\left(\mathbb{D},{ }^{4}\right.$ S. Coutiño de León $\mathbb{D},{ }^{10}$ E. De la Fuente $\mathbb{D},{ }^{11}$ R. Diaz Hernandez,${ }^{10}$ L. Diaz-Cruz, ${ }^{12}$ B.L. Dingus (D), ${ }^{1}$ M.A. DuVernois $\left(\mathbb{D},{ }^{13}\right.$ M. Durocher,${ }^{1}$ J.C. Díaz-Vélez (D), ${ }^{11}$ R.W. Ellsworth $\left(\mathbb{D},{ }^{9}\right.$ K. Engel (1D, ${ }^{9}$ C. Espinoza $\left(\mathbb{D},{ }^{2}\right.$ K.L. Fan,${ }^{9}$ K. Fang,${ }^{14}$ M. Fernández Alonso, ${ }^{6}$ H. Fleischhack $\left(\mathbb{D},{ }^{15}\right.$ N. Fraija ${ }^{16}$ A. Galván-Gámez, ${ }^{16}$ D. Garcia, ${ }^{2}$ J.A. García-González $\mathbb{D},{ }^{2}$ F. Garfias $\left(\mathbb{D},{ }^{16}\right.$ G. Giacinti,${ }^{17}$ M.M. González $\mathbb{D},{ }^{16}$ J.A. Goodman (D), ${ }^{9}$ J.P. Harding, ${ }^{1}$ S. Hernandez (iD, ${ }^{2}$ J. Hinton $\left(\mathbb{D},{ }^{17}\right.$ B. Hona, ${ }^{18}$ D. Huang $\left(\mathbb{D},{ }^{15}\right.$

F. Hueyotl-Zahuantitla $\mathbb{D},{ }^{3}$ P. Hüntemeyer,${ }^{15}$ A. Iriarte $\mathbb{D},{ }^{16}$ A. Jardin-Blice $\mathbb{D},{ }^{17,19,20}$ V. Joshi $\mathbb{D},{ }^{21}$ D. Kieda (D), ${ }^{18}$ A. Lara (D), ${ }^{5}$ W.H. Lee $\left(\mathbb{D},{ }^{16}\right.$ H. León Vargas $\left(\mathbb{D},{ }^{2}\right.$ C. DE León, ${ }^{4}$ J.T. Linnemann,${ }^{22}$ A.L. Longinotti $\left(\mathbb{D},{ }^{10,16}\right.$ G. Luis-Raya $\left(\mathbb{D},{ }^{23}\right.$ J. Lundeen, ${ }^{22}$ R. López-Coto,${ }^{24}$ K. Malone $\mathbb{D},{ }^{1}$ V. Marandon,${ }^{17}$ O. Martinez (Di), ${ }^{12}$ I. Martinez-Castellanos (D) ${ }^{9}$ J. Martínez-Castro, ${ }^{25}$ J.A. Matthews (D, ${ }^{26}$

P. Miranda-Romagnoli (iD, ${ }^{27}$ J.A. Morales-Soto, ${ }^{4}$ E. Moreno (iD, ${ }^{12}$ M. Mostafá (iD,${ }^{6}$ A. Nayerhoda (i),${ }^{7}$ L. Nellen $\mathbb{D},{ }^{28}$ M. Newbold $\mathbb{D},{ }^{18}$ M.U. Nisa $\mathbb{D},{ }^{22}$ R. Noriega-Papaqui $\left(\mathbb{D},{ }^{27}\right.$ L. Olivera-Nieto,,${ }^{17}$ N. Omodei $\left(\mathbb{D},{ }^{14}\right.$ A. Peisker, ${ }^{22}$ Y. Pérez Araujo (D), ${ }^{16}$ E.G. Pérez-Pérez (D), ${ }^{23}$ Z. Ren (D), ${ }^{26}$ C.D. Rho (D), ${ }^{29}$ C. Rivière (D) ${ }^{9}$ D. Rosa-González (DD, ${ }^{10}$ E. Ruiz-Velasco (D) ${ }^{17} \mathrm{H}$. Salazar, ${ }^{12} \mathrm{~F}$. Salesa Greus (D,,${ }^{7,30}$ A. Sandoval (D), ${ }^{2}$ M. Schneider (D), ${ }^{9}$ H. Schoorlemmer $\left(\mathbb{D},{ }^{17}\right.$ F. Serna,${ }^{2}$ G. Sinnis $\left(\mathbb{D},{ }^{1}\right.$ A.J. Smith,${ }^{9}$ R.W. Springer $(\mathbb{D}, 18$

P. Surajbali (1) ${ }^{17}$ K. Tollefson $\left(\mathbb{D},{ }^{22}\right.$ I. Torres $\left(\mathbb{D},{ }^{10}\right.$ R. Torres-Escobedo, ${ }^{11}$ T.N. Ukwatta $\left(\mathbb{D},{ }^{1}\right.$ F. Ureña-Mena, ${ }^{10}$ T. Weisgarber ${ }^{31}$ F. Werner (D), ${ }^{17}$ E. Willox, ${ }^{9}$ A. Zepeda (D ${ }^{32}$ And H. Zhou ${ }^{33}$

(HAWC COllaboration)

\footnotetext{
${ }^{1}$ Physics Division, Los Alamos National Laboratory, Los Alamos, NM, USA

${ }^{2}$ Instituto de Física, Universidad Nacional Autónoma de México, Ciudad de Mexico, Mexico

${ }^{3}$ Universidad Autónoma de Chiapas, Tuxtla Gutiérrez, Chiapas, México

${ }^{4}$ Universidad Michoacana de San Nicolás de Hidalgo, Morelia, Mexico

${ }^{5}$ Instituto de Geofísica, Universidad Nacional Autónoma de México, Ciudad de Mexico, Mexico

${ }^{6}$ Department of Physics, Pennsylvania State University, University Park, PA, USA

${ }^{7}$ Institute of Nuclear Physics Polish Academy of Sciences, PL-31342 IFJ-PAN, Krakow, Poland

${ }^{8}$ Department of Physics 83 Astronomy, University of Rochester, Rochester, NY, USA

${ }^{9}$ Department of Physics, University of Maryland, College Park, MD, USA

${ }^{10}$ Instituto Nacional de Astrofísica, Óptica y Electrónica, Puebla, Mexico

${ }^{11}$ Departamento de Física, Centro Universitario de Ciencias Exactase Ingenierias, Universidad de Guadalajara, Guadalajara, Mexico

${ }^{12}$ Facultad de Ciencias Físico Matemáticas, Benemérita Universidad Autónoma de Puebla, Puebla, Mexico

${ }^{13}$ Department of Physics and Wisconsin IceCube Particle Astrophysics Center, University of Wisconsin, University of Wisconsin-Madison, Madison, WI, USA

${ }^{14}$ Department of Physics, Stanford University: Stanford, CA 94305-4060, USA

${ }^{15}$ Department of Physics, Michigan Technological University, Houghton, MI, USA

${ }^{16}$ Instituto de Astronomía, Universidad Nacional Autónoma de México, Ciudad de Mexico, Mexico

${ }^{17}$ Max-Planck Institute for Nuclear Physics, 69117 Heidelberg, Germany

${ }^{18}$ Department of Physics and Astronomy, University of Utah, Salt Lake City, UT, USA
}

Corresponding author: Henrike Fleischhack

hfleisch@mtu.edu

Corresponding author: Mehr Un Nisa

nisamehr@msu.edu

Corresponding author: Alison Peisker

peiskera@msu.edu 


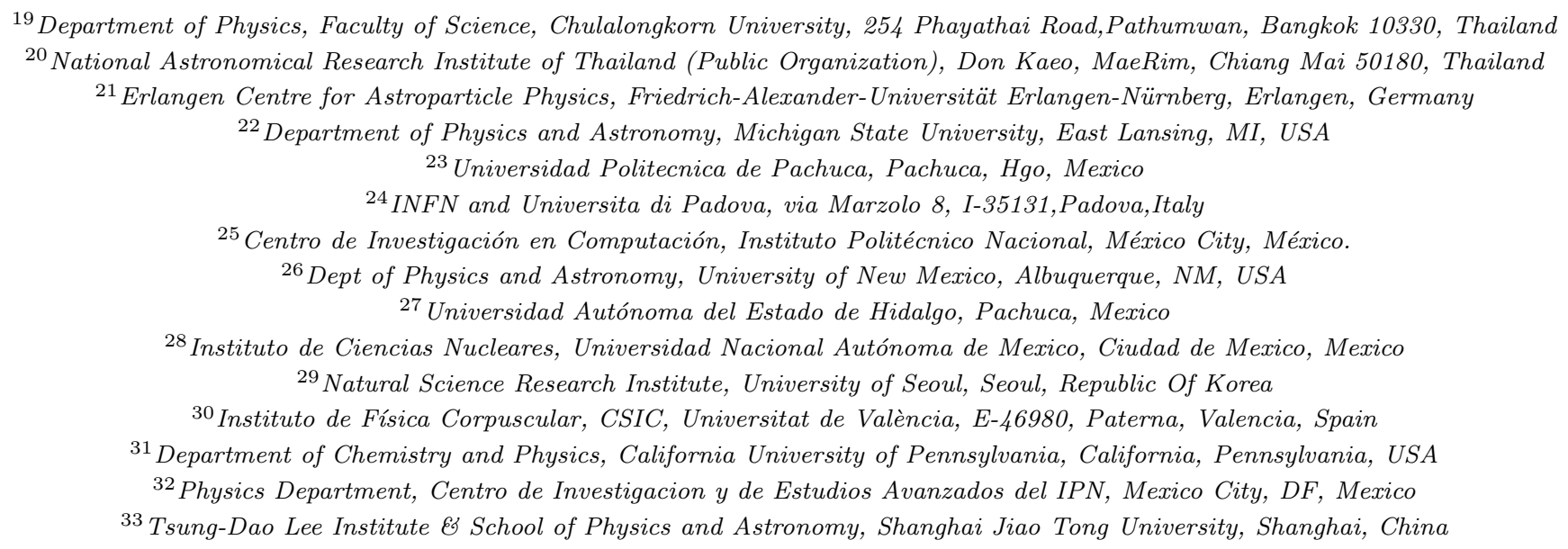

\section{ABSTRACT}

We present a new catalog of $\mathrm{TeV}$ gamma-ray sources using 1523 days of data from the High Altitude Water Cherenkov (HAWC) observatory. The catalog represents the most sensitive survey of the Northern gamma-ray sky at energies above several TeV, with three times the exposure compared to the previous HAWC catalog, 2 HWC. We report 65 sources detected at $\geq 5$ sigma significance, along with the positions and spectral fits for each source. The catalog contains eight sources that have no counterpart in the $2 \mathrm{HWC}$ catalog, but are within $1^{\circ}$ of previously detected $\mathrm{TeV}$ emitters, and twenty sources that are more than $1^{\circ}$ away from any previously detected $\mathrm{TeV}$ source. Of these twenty new sources, fourteen have a potential counterpart in the fourth Fermi Large Area Telescope catalog of gamma-ray sources. We also explore potential associations of $3 \mathrm{HWC}$ sources with pulsars in the ATNF pulsar catalog and supernova remnants in the Galactic supernova remnant catalog.

Keywords: High-energy astrophysics, Gamma-ray astronomy — catalogs — surveys

\section{INTRODUCTION}

High-sensitivity, unbiased surveys of the gamma-ray sky are important to finding new astrophysical objects - both to understand their bulk properties, and to constrain new physics beyond the standard model. Discovering the sources of cosmic rays and determining the underlying acceleration mechanisms requires precise measurements of gamma-ray spectra of objects above several tens of $\mathrm{TeV}$. In addition, the indirect search for dark matter particles in the GeV-TeV regime also hinges on detecting a steady flux of photons from several galactic and extra-galactic targets of interest. The current generation of ground-based gamma-ray telescopes, in particular Imaging Atmospheric Cherenkov Telescopes (Holder et al. 2009; Balzer et al. 2014; Ahnen et al. 2017; Anderhub et al. 2013), are capable of resolving gamma-ray sources to $\leq 0.1^{\circ}$ precision. The highly complementary survey instruments, such as Tibet-AS $\gamma$ (Amenomori et al. 2019), LHAASO (Zhen 2014), ARGO-YBJ (Bartoli et al. 2013), and HAWC, have further extended the reach of TeV astronomy with their high up-time and unprecedented sensitivity to spatially extended sources.

The High Altitude Water Cherenkov (HAWC) observatory has been continuously monitoring the Northern sky in $\mathrm{TeV}$ cosmic rays and gamma rays since commencing full operations in 2015, and has achieved a sensitivity down to a few percent of the Crab flux in five years. This work presents the results of an all-sky time-integrated search for pointlike and extended sources using 1523 days of HAWC data. As a follow-up to the $2 \mathrm{HWC}$ catalog of TeV gamma-ray sources (Abeysekara et al. 2017b), we introduce an updated catalog using more data and improved analysis methods.

This paper is structured as follows. Section 2 provides a brief description of the HAWC detector, as well as the data and the analysis method we use in the construction of the catalog. Section 3 presents the results of the catalog search and provides preliminary spatial and spectral information on all sources. A broad discussion of the results is also presented. Section 4 discusses the systematic uncertainties and methodological limitations of this work. Section 5 concludes the paper.

\section{INSTRUMENT AND DATA ANALYSIS}




\subsection{The HAWC Gamma-ray Observatory}

HAWC consists of 300 water tanks, each filled with $~ 200,000$ liters of purified water and instrumented with four photo-multiplier tubes (PMTs). Very-high-energy primary particles interact with Earth's atmosphere and create an extensive air shower of secondary particles. Charged particles produced in an air shower emit Cherenkov radiation as they pass through the water in HAWC's tanks, which in turn produces photo-electrons in the PMTs.

The HAWC observatory is sensitive to gamma rays in an energy range from hundreds of GeV to hundreds of TeV, with the exact energy threshold depending on the declination and energy spectrum of each source. Due to its location at a latitude of $19^{\circ} \mathrm{N}$ and its wide field-of-view, HAWC can observe about two thirds of the gamma-ray sky (from about $-26^{\circ}$ to $+64^{\circ}$ in declination) every day, with an instantaneous field-of-view of $>2.0$ sr. HAWC's angular resolution (68\% containment radius for photons) ranges between $0.1^{\circ}$ and $1.0^{\circ}$ depending on the energy and zenith angle of the signal. More details about the HAWC detector can be found in Abeysekara et al. (2017b,c).

\subsection{Data Selection and Reduction}

The temporal and spatial distribution of charge deposited in HAWC's PMTs is used to reconstruct the properties of the primary particle producing the air shower. The difference in timing between the signals recorded in different tanks allows us to reconstruct the direction of the primary particle. The spatial distribution and magnitude of the charges can be used to reconstruct the primary energy and to separate gamma-ray induced showers from cosmic-ray induced ones.

We distribute the reconstructed events into nine analysis bins according to the fraction of the operating PMTs that recorded a signal for a given event. The fraction of PMTs hit is correlated with the primary energy, allowing us to extract the energy spectrum of gamma-ray sources. We apply gamma-hadron separation cuts to reduce the background of cosmic-ray induced showers. A detailed description of air shower reconstructions and quality cuts applied to the data can be found in Abeysekara et al. (2017b,c).

We further bin the gamma-ray candidate events according to the direction of the primary particle in celestial coordinates (Right Ascension and Declination, J2000 epoch). We use the HEALPix binning scheme (Gorski et al. 2005), with an NSIDE parameter of 1024.

The dominant background is given by hadronic showers that pass the gamma-hadron cuts. For each pixel, we estimate the expected number of remaining background events after cuts using the method of Direct Integration as described in Atkins et al. (2003).

\subsection{Construction of the Catalog}

The 3HWC catalog is based on data collected by the HAWC observatory between November 2014 and June 2019, corresponding to a livetime of 1523 days - about three times the livetime of the $2 \mathrm{HWC}$ catalog data set. For the most part, the construction of the catalog follows the same method as the previous $2 \mathrm{HWC}$ catalog, which is described in Abeysekara et al. (2017b). We summarize the algorithm below, with particular focus on the differences from the previous catalog search.

\subsubsection{Source Search}

We perform a blind search for sources across HAWC's field-of-view using the likelihood framework discussed in Younk et al. (2015). The likelihood calculation assumes that the number of counts in each bin/pixel is distributed according to a Poisson distribution, with the mean given by the estimated background counts plus (if applicable) the predicted number of gamma-ray counts from the convolution of the source model with the detector response.

For each HEALPix pixel, we calculate a likelihood ratio $\lambda=\hat{\mathcal{L}}_{s+b} / \mathcal{L}_{b}$, comparing the likelihood, $\hat{\mathcal{L}}_{s+b}$, of the best-fit model with a gamma-ray source centered on that pixel to that of a background-only model, $\mathcal{L}_{b}$. We define a test statistic, $T S=2 \log (\Lambda)$. Assuming that the null hypothesis is true, the $T S$ is distributed according to a $\chi^{2}$ distribution with one degree of freedom (Wilks 1938), which can be approximated by a gaussian distribution. Then, $\pm \sqrt{T S}$ corresponds to the ("pre-trials") significance. The negative sign is used for pixels in which the best-fit flux normalization is negative.

The signal hypothesis considers a fixed source morphology and an $E^{-2.5}$ power-law energy spectrum. The only free parameter of the likelihood fit is the flux normalization. We repeat source searches for four different hypothetical morphologies: point sources, and extended disk-like sources with radii of $0.5^{\circ}, 1.0^{\circ}$, and $2.0^{\circ}$. This procedure is very similar to what was used in the previous $2 \mathrm{HWC}$ catalog, with the only change being the spectral index hypothesis 
(the prior catalog used a spectral index of -2.7 for point sources, -2.0 for extended sources). The resulting all-sky significance map for a point-source assumption can be seen in Figure 1.

For each significance map, we compile a list of candidate sources comprising the local maxima with $\sqrt{T S}>5$. Due to Poisson fluctuations in the number of detected gamma rays, a single source may produce multiple local maxima. To avoid double-counting such sources, candidate sources are promoted to sources if they pass the following "TS valley" criterion: the significance profile connecting the source candidate with any other source within $5^{\circ}$ of the source candidate in question has to "dip" by $\Delta T S>2$ to promote a source candidate to a "primary" source status. A "secondary" source is defined with a relaxed criterion, such that $1<\Delta T S<2$. We mark secondary sources with a dagger ( $\dagger$ ) in Table 1 . The $\Delta T S$ criterion used for $3 \mathrm{HWC}$ is a little stricter than the one used in the $2 \mathrm{HWC}$ catalog, in which a source only had to pass the $\Delta T S$ with its closest neighboring source.

We then combine the four source lists (for the four different assumptions of the source morphology) to yield the $3 \mathrm{HWC}$ catalog. We include all sources found in the point source search in $3 \mathrm{HWC}$. We only include sources found in the extended source searches if they are more than $1^{\circ}$ away from any point source or smaller extended source already in the catalog. Table 1 shows the resulting list of sources comprising the $3 \mathrm{HWC}$ catalog. For each source, we also show the closest known $\mathrm{TeV}$ source listed in the $\mathrm{TeVCat}^{1}$ (Wakely \& Horan 2008) if it is within $1^{\circ}$ of the HAWC source.

\subsubsection{Spectral Fits}

After identifying the primary and secondary sources, we perform likelihood fits to obtain each source's energy spectrum. We assume a simple power-law spectrum for each source, fitting for the spectral index and flux normalization as done for the $2 \mathrm{HWC}$ catalog (Abeysekara et al. 2017b). There are two changes to the spectral fitting procedure used in this work compared to $2 \mathrm{HWC}$. First, for $3 \mathrm{HWC}$, we only report the spectral fit for the same morphology for which the source was first found in the source-search stage. Second, we dynamically treat the instrument response during the fit. 2HWC fits relied on a method where the angular resolution was pre-calculated (and fit with a double-Gaussian function in each analysis bin) before the spectral fit, for a fixed spectral assumption. 3HWC spectral fits use a new method in which we recalculate the angular resolution for each tested spectral assumption during the fit. Additionally, we do not assume that the angular resolution follows a specific analytical shape. This allows for a more complete
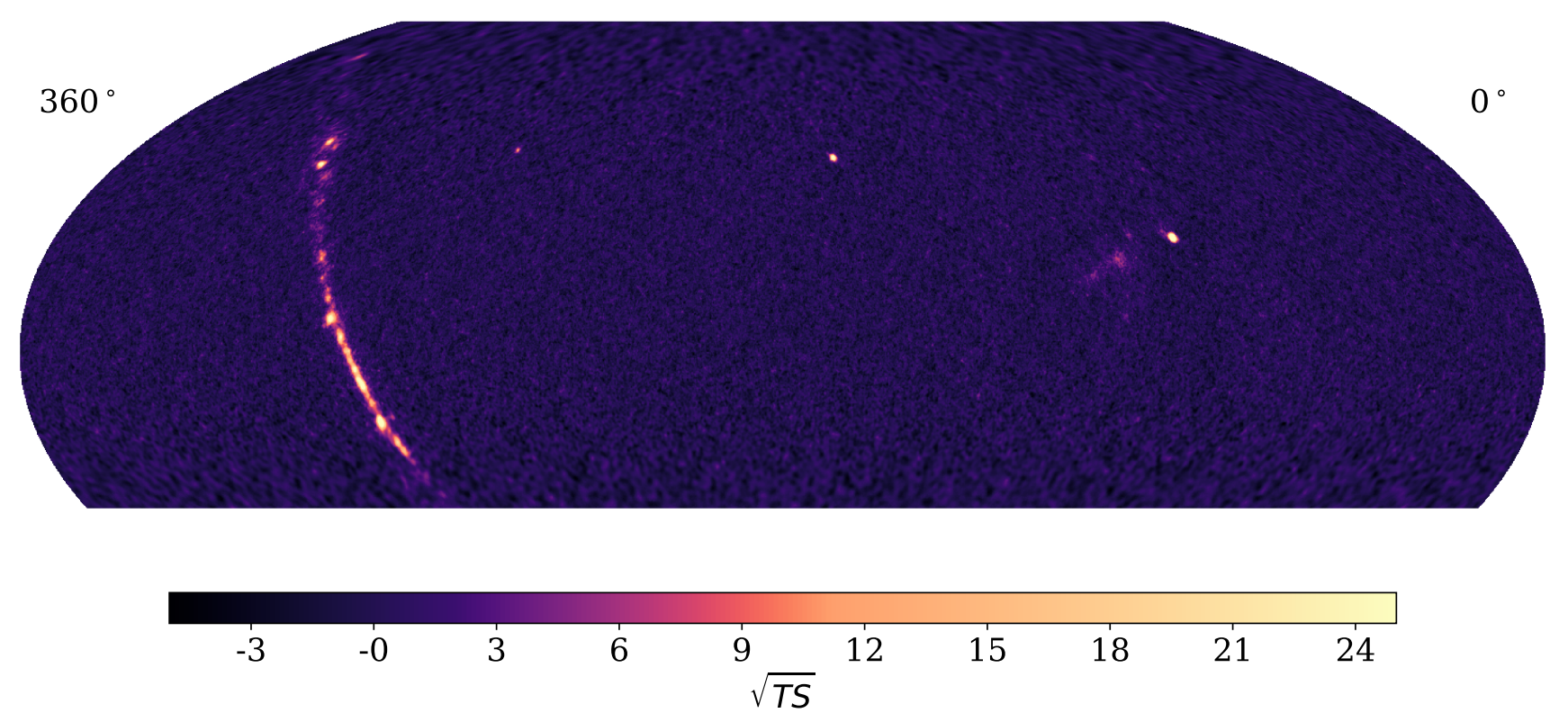

Figure 1. All-sky significance map in celestial coordinates, assuming a point-source hypothesis. The bright band on the left is part of the Galactic plane (c.f. Figures 4-7), and the bright region on the right is the Galactic anti-center region containing the Crab Nebula and the Geminga halo (c.f. Figure 3). The two off-plane hotspots are the two TeV-bright blazars Mrk 421 (right) and Mrk 501 (left).

${ }^{1}$ http://tevcat.uchicago.edu/ 
characterization of HAWC's PSF and of the systematic uncertainties on the fit parameters. See Martinez-Castellanos (2019) for more details on the new spectral fit method. Table 2 shows the results of the spectral fits.

\section{RESULTS}

\section{1. $3 H W C$ Sources}

The 3HWC catalog contains 65 sources, 17 of which are considered secondary sources (not well separated from neighboring sources according to the $\Delta T S$ criterion). The source positions can be found in Table 1 and the results of the spectral fits, as well as the energy range from which we expect $75 \%$ of the observed significance, can be found in Table 2. Twenty-eight of these sources do not lie within $1^{\circ}$ of any $2 \mathrm{HWC}$ source. We discuss some of these sources in more detail in Section 3.4.

We compare the flux measurements with the sensitivity for the underlying dataset. The flux sensitivity is defined as the flux normalization required to have a $50 \%$ probability of detecting a source at the $5 \sigma$ level. Figure 2 shows the HAWC 1523-days sensitivity and the flux measurements from Table 2 as a function of declination. HAWC is more sensitive to sources transiting directly overhead, corresponding to a declination of $19.0^{\circ}$, than to sources transiting at larger zenith angles. HAWC is also more sensitive to hard-spectrum sources. For the optimal case (an $E^{-2}$ source transiting directly overhead), HAWC's sensitivity approaches $\sim 2 \%$ of the flux of the Crab Nebula. The sensitivity is nearly constant with respect to the Right Ascension of a source (it varies by less than $3 \%$ across the sky).

Most of the sources were found in the point source search. With about three times the livetime compared to the $2 \mathrm{HWC}$ catalog, many extended sources are now also significantly detected in the point source map. For example, Figure 3 shows five 3 HWC sources (3HWC J0630+186, 3HWC J0631+169, 3HWC J0633+191, 3HWC J0634+165, and $\mathbf{3 H W C} \mathbf{J 0 6 3 4}+\mathbf{1 8 0}$, all found in the point source search) clustering near the Geminga pulsar. We believe that these five sources are all part of the extended halo around Geminga, described in Abeysekara et al. (2017a). Similarly,

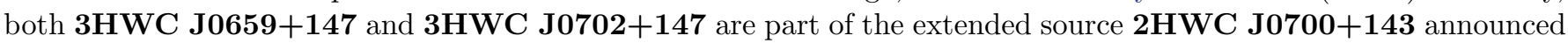
in the aforementioned publication. It is not clear if these sources correspond to real features in the morphology of the two pulsar halos, or if they are just due to statistical fluctuations in the number of photons recorded by HAWC.

As seen in the the all-sky significance map (Figure 1), the majority of the sources in the 3HWC catalog are located along the Galactic plane. Figures 4, 5, 6, and 7 show the significance maps of the Galactic plane from the Cygnus region $\left(\mathrm{l}=85^{\circ}\right)$ to the inner Galaxy $\left(\mathrm{l}=2^{\circ}\right)$. The Galactic center itself falls outside of the part of the sky visible to HAWC. Figure 3 shows a region near the Galactic anti-center containing the Crab Nebula, Geminga, and other sources. For this region, both the point-source significance map and the significance map from the $1^{\circ}$ extended source search are shown. For convenience, the locations of 3HWC sources and TeVCat sources have been marked in these images. Figures 8 and 9 show the distribution of 3HWC sources as a function of galactic latitude and longitude respectively. 
Table 1. Source list and nearest TeVCat sources (within $1^{\circ}$ of each 3 HWC source). Secondary sources (i.e., sources that are not separated from their neighbor(s) by a large TS gap) are marked with a dagger ( $\dagger$ ). The position uncertainty reported here is statistical only. The systematic uncertainty on the position is discussed in Section 4.3. TeVCat source names within $0.5^{\circ}$ of a $3 \mathrm{HWC}$ source are printed in bold. For sources without a TeVCat counterpart within $1^{\circ}$, the angular distance to the nearest TeVCat source is printed for reference.

\begin{tabular}{|c|c|c|c|c|c|c|c|c|c|}
\hline \multirow[b]{2}{*}{ Name } & \multirow[b]{2}{*}{$\begin{array}{c}\text { Radius } \\
{\left[^{\circ}\right]}\end{array}$} & \multirow[b]{2}{*}{$\mathrm{TS}$} & \multirow[b]{2}{*}{$\begin{array}{c}\mathrm{RA} \\
\left.{ }^{\circ}\right] \\
\end{array}$} & \multirow[b]{2}{*}{$\begin{array}{c}\text { Dec } \\
{\left[{ }^{\circ}\right]} \\
\end{array}$} & \multirow[b]{2}{*}{$\begin{array}{c}l \\
{\left[{ }^{\circ}\right]} \\
\end{array}$} & \multirow[b]{2}{*}{$\begin{array}{c}b \\
\left.{ }^{\circ}\right] \\
\end{array}$} & \multirow[b]{2}{*}{$1 \sigma$ stat. unc. } & \multicolumn{2}{|c|}{ Nearest TeVCat source } \\
\hline & & & & & & & & $\begin{array}{c}\text { Dist. } \\
{\left[^{\circ}\right]}\end{array}$ & Name \\
\hline $3 \mathrm{HWC} \mathrm{J0534+220}$ & 0.0 & 35736.5 & 83.63 & 22.02 & 184.55 & -5.78 & 0.06 & 0.01 & Crab \\
\hline $3 \mathrm{HWC}$ J0540+228 ${ }^{\dagger}$ & 0.0 & 28.8 & 85.17 & 22.87 & 184.58 & -4.13 & 0.11 & 0.77 & HAWC J0543+233 \\
\hline 3HWC J0543+231 & 0.0 & 34.2 & 85.78 & 23.11 & 184.67 & -3.52 & 0.10 & 0.29 & HAWC J0543+233 \\
\hline 3 HWC J0617+224 & 0.0 & 32.3 & 94.39 & 22.47 & 189.18 & 3.05 & 0.14 & 0.17 & IC 443 \\
\hline 3 HWC J0621+382 & 0.5 & 28.0 & 95.32 & 38.21 & 175.44 & 10.97 & 0.30 & 14.56 & $\ldots$ \\
\hline $3 \mathrm{HWC}$ J0630+186 & 0.0 & 38.9 & 97.69 & 18.68 & 193.98 & 4.02 & 0.10 & 1.18 & $\ldots$ \\
\hline 3HWC J0631+107 & 0.0 & 26.5 & 97.78 & 10.73 & 201.08 & 0.43 & 0.09 & 3.84 & $\ldots$ \\
\hline $3 \mathrm{HWC}$ J0631+169 ${ }^{\dagger}$ & 0.0 & 39.1 & 97.95 & 16.96 & 195.63 & 3.45 & 0.17 & 0.44 & Geminga \\
\hline 3HWC J0633+191 & 0.0 & 27.5 & 98.44 & 19.12 & 193.92 & 4.85 & 0.28 & 1.35 & $\ldots$ \\
\hline 3HWC J0634+067 & 0.5 & 36.2 & 98.66 & 6.73 & 205.03 & -0.65 & 0.22 & 0.28 & HAWC J0635+070 \\
\hline $3 \mathrm{HWC} \mathrm{J} 0634+165^{\dagger}$ & 0.0 & 30.0 & 98.53 & 16.53 & 196.26 & 3.74 & 0.11 & 0.92 & Geminga \\
\hline 3HWC J0634+180 & 0.0 & 60.0 & 98.75 & 18.05 & 195.00 & 4.62 & 0.09 & 0.38 & Geminga Pulsar \\
\hline $3 \mathrm{HWC} \mathrm{J0659}+147^{\dagger}$ & 0.0 & 28.8 & 104.85 & 14.75 & 200.60 & 8.40 & 0.12 & 0.50 & $2 \mathrm{HWC} \mathrm{J0700+143}$ \\
\hline 3HWC J0702+147 & 0.0 & 28.9 & 105.56 & 14.75 & 200.91 & 9.01 & 0.19 & 0.60 & 2HWC J0700+143 \\
\hline 3HWC J1104+381 & 0.0 & 3025.3 & 166.11 & 38.16 & 179.95 & 65.05 & 0.06 & 0.04 & Markarian 421 \\
\hline 3HWC J1654+397 & 0.0 & 227.8 & 253.52 & 39.74 & 63.58 & 38.82 & 0.09 & 0.05 & Markarian 501 \\
\hline 3HWC J1739+099 & 0.0 & 28.2 & 264.99 & 9.93 & 33.89 & 20.34 & 0.06 & 2.87 & $\ldots$ \\
\hline 3HWC J1743+149 & 0.0 & 25.9 & 265.82 & 14.94 & 39.13 & 21.68 & 0.10 & 4.61 & $\ldots$ \\
\hline 3HWC J1757-240 & 1.0 & 28.6 & 269.30 & -24.09 & 5.49 & 0.25 & 0.48 & 0.74 & HESS J1800-240B \\
\hline $3 \mathrm{HWC} \mathrm{J} 1803-211^{\dagger}$ & 0.0 & 38.4 & 270.97 & -21.18 & 8.78 & 0.36 & 0.27 & 0.54 & HESS J1804-216 \\
\hline 3HWC J1809-190 & 0.0 & 264.8 & 272.46 & -19.04 & 11.33 & 0.18 & 0.08 & 0.31 & HESS J1809-193 \\
\hline 3HWC J1813-125 & 0.0 & 51.9 & 273.34 & -12.52 & 17.46 & 2.57 & 0.14 & 0.17 & HESS J1813-126 \\
\hline 3HWC J1813-174 & 0.0 & 416.0 & 273.43 & -17.47 & 13.15 & 0.13 & 0.06 & 0.18 & 2HWC J1814-173 \\
\hline $3 \mathrm{HWC} \mathrm{J} 1819-150^{\dagger}$ & 0.0 & 93.8 & 274.79 & -15.09 & 15.86 & 0.11 & 0.14 & 0.05 & 2HWC J1819-150* \\
\hline 3HWC J1825-134 & 0.0 & 2212.5 & 276.46 & -13.40 & 18.12 & -0.53 & 0.06 & 0.00 & 2HWC J1825-134 \\
\hline 3HWC J1831-095 & 0.0 & 237.7 & 277.87 & -9.59 & 22.13 & 0.02 & 0.14 & 0.31 & HESS J1831-098 \\
\hline 3HWC J1837-066 & 0.0 & 1542.7 & 279.40 & -6.62 & 25.47 & 0.04 & 0.06 & 0.06 & 2HWC J1837-065 \\
\hline 3HWC J1843-034 & 0.0 & 876.6 & 280.99 & -3.47 & 28.99 & 0.08 & 0.06 & 0.24 & 2HWC J1844-032 \\
\hline $3 \mathrm{HWC}$ J1844-001 ${ }^{\dagger}$ & 0.0 & 33.2 & 281.07 & -0.19 & 31.95 & 1.50 & 0.15 & 1.20 & $\ldots$ \\
\hline 3HWC J1847-017 & 0.0 & 338.1 & 281.95 & -1.75 & 30.96 & 0.01 & 0.09 & 0.17 & HESS J1848-018 \\
\hline 3HWC J1849+001 & 0.0 & 427.5 & 282.35 & 0.15 & 32.83 & 0.52 & 0.06 & 0.16 & IGR J18490-0000 \\
\hline $3 \mathrm{HWC} \mathrm{J} 1852+013^{\dagger}$ & 0.0 & 126.7 & 283.05 & 1.34 & 34.21 & 0.44 & 0.09 & 0.06 & $2 \mathrm{HWC} \mathrm{J1852+013^{* }}$ \\
\hline 3HWC J1857+027 & 0.0 & 763.5 & 284.33 & 2.80 & 36.09 & -0.03 & 0.06 & 0.14 & HESS J1857+026 \\
\hline 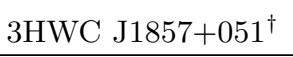 & 0.0 & 35.3 & 284.33 & 5.19 & 38.22 & 1.06 & 0.11 & 1.23 & $\ldots$ \\
\hline
\end{tabular}

Table 1 continued on next page 
Table 1 (continued)

\begin{tabular}{|c|c|c|c|c|c|c|c|c|c|}
\hline \multirow[b]{2}{*}{ Name } & \multirow[b]{2}{*}{$\begin{array}{c}\text { Radius } \\
{\left[^{\circ}\right]}\end{array}$} & \multirow[b]{2}{*}{$\mathrm{TS}$} & \multirow[b]{2}{*}{$\begin{array}{l}\mathrm{RA} \\
\left.{ }^{\circ}\right] \\
\end{array}$} & \multirow[b]{2}{*}{$\begin{array}{l}\text { Dec } \\
{\left[^{\circ}\right]}\end{array}$} & \multirow[b]{2}{*}{$\begin{array}{c}l \\
{\left[^{\circ}\right]} \\
\end{array}$} & \multirow[b]{2}{*}{$\begin{array}{c}b \\
{\left[^{\circ}\right]}\end{array}$} & \multirow[b]{2}{*}{$1 \sigma$ stat. unc. } & \multicolumn{2}{|c|}{ Nearest TeVCat source } \\
\hline & & & & & & & & $\begin{array}{l}\text { Dist. } \\
{\left[^{\circ}\right]}\end{array}$ & Name \\
\hline $3 \mathrm{HWC}$ J1907+085 & 0.0 & 75.5 & 286.79 & 8.57 & 42.35 & 0.44 & 0.09 & 0.07 & 2HWC J1907+084* \\
\hline 3HWC J1908+063 & 0.0 & 1320.9 & 287.05 & 6.39 & 40.53 & -0.80 & 0.06 & 0.14 & MGRO J1908+06 \\
\hline 3HWC J1912+103 & 0.0 & 198.2 & 288.06 & 10.35 & 44.50 & 0.15 & 0.09 & 0.24 & HESS J1912+101 \\
\hline $3 \mathrm{HWC} \mathrm{J} 1913+048^{\dagger}$ & 0.0 & 44.7 & 288.32 & 4.86 & 39.75 & -2.63 & 0.13 & 0.11 & SS 433 e1 \\
\hline 3HWC J1914+118 & 0.0 & 102.9 & 288.68 & 11.87 & 46.13 & 0.32 & 0.09 & 0.15 & $2 \mathrm{HWC} \mathrm{J1914+117*}$ \\
\hline $3 \mathrm{HWC}$ J1915+164 & 0.0 & 27.5 & 288.76 & 16.41 & 50.19 & 2.35 & 0.10 & 2.92 & $\ldots$ \\
\hline 3HWC J1918+159 & 0.0 & 31.6 & 289.69 & 15.91 & 50.16 & 1.33 & 0.11 & 1.99 & $\ldots$ \\
\hline $3 \mathrm{HWC} \mathrm{J} 1920+147^{\dagger}$ & 0.0 & 55.4 & 290.17 & 14.79 & 49.39 & 0.39 & 0.10 & 0.80 & $\mathrm{~W} 51$ \\
\hline $3 \mathrm{HWC}$ J1922+140 & 0.0 & 176.6 & 290.70 & 14.09 & 49.01 & -0.38 & 0.06 & 0.10 & W 51 \\
\hline $3 \mathrm{HWC} \mathrm{J} 1923+169^{\dagger}$ & 0.0 & 46.1 & 290.79 & 16.96 & 51.58 & 0.89 & 0.10 & 1.54 & $\ldots$ \\
\hline 3HWC J1928+178 & 0.0 & 216.7 & 292.10 & 17.82 & 52.93 & 0.20 & 0.08 & 0.06 & $2 \mathrm{HWC} \mathrm{J1928+177}$ \\
\hline 3HWC J1930+188 & 0.0 & 115.6 & 292.54 & 18.84 & 54.03 & 0.32 & 0.09 & 0.09 & SNR G054.1+00.3 \\
\hline $3 \mathrm{HWC} \mathrm{J} 1935+213^{\dagger}$ & 0.0 & 26.2 & 293.95 & 21.38 & 56.90 & 0.39 & 0.12 & 1.89 & $\ldots$ \\
\hline $3 \mathrm{HWC}$ J1936+223 & 0.0 & 28.2 & 294.08 & 22.31 & 57.76 & 0.73 & 0.11 & 1.62 & $\ldots$ \\
\hline $3 \mathrm{HWC}$ J1937+193 & 0.0 & 25.2 & 294.39 & 19.31 & 55.29 & -0.98 & 0.13 & 1.72 & $\ldots$ \\
\hline $3 \mathrm{HWC} \mathrm{J} 1940+237$ & 0.0 & 27.2 & 295.05 & 23.77 & 59.47 & 0.67 & 0.36 & 0.28 & 2HWC J1938+238 \\
\hline 3HWC J1950+242 & 0.0 & 25.1 & 297.69 & 24.26 & 61.10 & -1.16 & 0.11 & 0.32 & $2 \mathrm{HWC} \mathrm{J1949+244}$ \\
\hline $3 \mathrm{HWC} \mathrm{J} 1951+266^{\dagger}$ & 0.5 & 35.6 & 297.90 & 26.61 & 63.23 & -0.13 & 0.61 & 2.14 & $\ldots$ \\
\hline $3 \mathrm{HWC}$ J1951+293 & 0.0 & 68.7 & 297.99 & 29.40 & 65.66 & 1.23 & 0.14 & 0.25 & $2 \mathrm{HWC} \mathrm{J1953+294}$ \\
\hline $3 \mathrm{HWC} \mathrm{J} 1954+286^{\dagger}$ & 0.0 & 48.3 & 298.70 & 28.63 & 65.32 & 0.30 & 0.14 & 0.12 & $2 \mathrm{HWC} \mathrm{J1955+285}$ \\
\hline $3 \mathrm{HWC} \mathrm{J1957+291}$ & 0.0 & 41.0 & 299.36 & 29.18 & 66.09 & 0.10 & 0.10 & 0.75 & 2HWC J1955+285 \\
\hline $3 \mathrm{HWC}$ J2005+311 & 0.0 & 33.1 & 301.46 & 31.17 & 68.74 & -0.40 & 0.12 & 3.01 & $\ldots$ \\
\hline $3 \mathrm{HWC}$ J2006+340 & 0.0 & 67.4 & 301.73 & 34.00 & 71.25 & 0.94 & 0.14 & 0.23 & 2HWC J2006+341 \\
\hline $3 \mathrm{HWC} \mathrm{J} 2010+345^{\dagger}$ & 0.0 & 27.6 & 302.69 & 34.55 & 72.14 & 0.56 & 0.17 & 1.01 & $\ldots$ \\
\hline 3HWC J2019+367 & 0.0 & 1227.5 & 304.94 & 36.80 & 75.02 & 0.30 & 0.06 & 0.07 & VER J2019+368 \\
\hline 3HWC J2020+403 & 0.0 & 93.9 & 305.16 & 40.37 & 78.07 & 2.19 & 0.09 & 0.40 & VER J2019+407 \\
\hline 3 HWC J2022+431 & 0.0 & 29.0 & 305.52 & 43.16 & 80.52 & 3.54 & 0.10 & 1.80 & $\ldots$ \\
\hline 3HWC J2023+324 & 1.0 & 30.7 & 305.81 & 32.44 & 71.85 & -2.77 & 0.73 & 3.96 & $\ldots$ \\
\hline $3 \mathrm{HWC}$ J2031+415 & 0.0 & 556.9 & 307.93 & 41.51 & 80.21 & 1.14 & 0.06 & 0.07 & TeV J2032+4130 \\
\hline $3 \mathrm{HWC} \mathrm{J} 2043+443^{\dagger}$ & 0.5 & 28.6 & 310.89 & 44.30 & 83.74 & 1.10 & 0.24 & 2.88 & $\ldots$ \\
\hline $3 \mathrm{HWC}$ J2227+610 & 0.0 & 52.5 & 336.96 & 61.05 & 106.42 & 2.87 & 0.19 & 0.16 & Boomerang \\
\hline
\end{tabular}


Table 2. Source radius, best-fit spectrum, and energy range. The flux $F_{7}$ is the differential flux at $7 \mathrm{TeV}$. The two sets of reported uncertainties correspond to statistical and systematic, respectively. The spectral fit for 3HWC J0659+147 did not converge. The energy range quoted here is the true energy interval from which we expect to get $75 \%$ of a given source's significance.

\begin{tabular}{|c|c|c|c|c|}
\hline Name & $\begin{array}{l}\text { Radius } \\
\left.\qquad{ }^{\circ}\right]\end{array}$ & Index & $\begin{array}{c}F_{7} \\
{\left[10^{-15} \mathrm{TeV}^{-1} \mathrm{~cm}^{-2} \mathrm{~s}^{-1}\right]}\end{array}$ & $\begin{array}{c}\text { Energy Range } \\
\qquad[\mathrm{TeV}]\end{array}$ \\
\hline 3 HWC J0534+220 & 0.0 & $-2.579 \pm 0.005_{-0.018}^{+0.067}$ & $234.2 \pm 1.4_{-34.0}^{+55.2}$ & $1.6-37.4$ \\
\hline $3 \mathrm{HWC}$ J0540+228 & 0.0 & $-2.84 \pm 0.14_{-0.03}^{+0.14}$ & $4.8 \stackrel{+0.7}{+0.8} \stackrel{-0.8}{-1.1}_{-0.8}$ & $1.1-28.5$ \\
\hline $3 \mathrm{HWC}$ J0543+231 & 0.0 & $-2.13_{-0.16}^{+0.15}{ }_{-0.01}^{+0.16}$ & $4.2 \pm 0.9_{-0.6}^{+1.9}$ & $10.5-205.9$ \\
\hline 3 HWC J0617+224 & 0.0 & $-3.05 \pm 0.11_{-0.02}^{+0.06}$ & $4.5 \pm 0.8_{-0.8}^{+1.2}$ & $0.5-12.0$ \\
\hline 3 HWC J0621+382 & 0.5 & $-2.41_{-0.13}^{+0.12} \underset{-0.01}{+0.08}$ & $8.9_{-1.5}^{+1.4} \stackrel{-1.2}{+2.6}^{+2.6}$ & $5.7-138.3$ \\
\hline $3 \mathrm{HWC}$ J0630+186 & 0.0 & $-2.21_{-0.14}^{+0.13} \stackrel{-0.02}{+0.14}^{+0.02}$ & $5.1+0.9+2.8$ & $8.4-183.8$ \\
\hline 3 HWC J0631+107 & 0.0 & $-2.23_{-0.19}^{+0.17}{ }_{-0.01}^{+0.10}$ & $4.0 \stackrel{+0.9+1.4}{+0.4}$ & $8.6-186.9$ \\
\hline 3HWC J0631+169 & 0.0 & $-2.51{ }_{-0.14}^{+0.13}{ }_{-0.03}^{+0.13}$ & $5.9 \pm 0.7_{-0.9}^{+2.0}$ & $3.3-95.0$ \\
\hline 3HWC J0633+191 & 0.0 & $-2.64{ }_{-0.15}^{+0.14}{ }_{-0.02}^{+0.10}$ & $4.8 \pm 0.7_{-0.7}^{+1.3}$ & $2.1-61.9$ \\
\hline 3HWC J0634+067 & 0.5 & $-2.27 \pm 0.10_{-0.01}^{+0.09}$ & $9.0_{-1.4}^{+1.3} \stackrel{-1.2}{+2.7}^{+2.7}$ & $7.6-171.1$ \\
\hline 3HWC J0634+165 & 0.0 & $-2.52{ }_{-0.22}^{+0.19}{ }_{-0.03}^{+0.13}$ & $5.0{ }_{-0.8}^{+0.7}+1.6$ & $3.2-93.0$ \\
\hline 3HWC J0634+180 & 0.0 & $-2.47_{-0.11}^{+0.10} \stackrel{-0.02}{0.11}_{-0.02}$ & $7.3 \pm 0.7_{-1.1}^{+2.2}$ & $3.7-102.0$ \\
\hline $3 \mathrm{HWC}$ J0659+147 & 0.0 & $\ldots$ & $\ldots$ & $\ldots$ \\
\hline 3 HWC J0702+147 & 0.0 & $-1.99{ }_{-0.24}^{+0.19}{ }_{-0.02}^{+0.18}$ & $3.6{ }_{-1.3}^{+1.1} \stackrel{-0.5}{+1.9}_{0}$ & $14.1-261.8$ \\
\hline 3HWC J1104+381 & 0.0 & $-3.04 \pm 0.01{ }_{-0.02}^{+0.06}$ & $69.4 \pm 1.3_{-11.4}^{+16.6}$ & $0.7-15.0$ \\
\hline 3HWC J1654+397 & 0.0 & $-2.91 \pm 0.044_{-0.02}^{+0.06}$ & $20.0 \pm 1.0_{-3.2}^{+5.3}$ & $1.3-29.4$ \\
\hline 3HWC J1739+099 & 0.0 & $-1.98{ }_{-0.14}^{+0.13}{ }_{-0.02}^{+0.12}$ & $3.3 \pm 0.8_{-0.6}^{+1.4}$ & $14.8-274.0$ \\
\hline $3 \mathrm{HWC}$ J1743+149 & 0.0 & $-2.37_{-0.16}^{+0.15} \stackrel{-0.02}{+0.08}^{+0.08}$ & $4.0 \stackrel{+0.7}{+0.8} \stackrel{+1.3}{-0.6}$ & $5.6-139.3$ \\
\hline 3HWC J1757-240 & 1.0 & $-2.80 \pm 0.11_{-0.03}^{+0.07}$ & $72.0{ }_{-11.3}^{+10.9}{ }_{-9.2}^{+17.6}$ & $5.6-158.4$ \\
\hline 3HWC J1803-211 & 0.0 & $-2.59{ }_{-0.14}^{+0.13} \stackrel{-0.04}{+0.09}^{+0.09}$ & $27.9 \stackrel{+5.4}{+5.40 .3}$ & $9.7-206.8$ \\
\hline 3HWC J1809-190 & 0.0 & $-2.59 \pm 0.05_{-0.03}^{+0.09}$ & $68.0_{-4.5}^{+4.4}{ }_{-9.7}^{+24.5}$ & $7.7-177.3$ \\
\hline 3HWC J1813-125 & 0.0 & $-2.81 \pm 0.10_{-0.03}^{+0.09}$ & $19.9 \pm 2.0_{-3.1}^{+5.9}$ & $2.6-69.2$ \\
\hline 3HWC J1813-174 & 0.0 & $-2.54 \pm 0.044_{-0.02}^{+0.09}$ & $74.0 \pm 4.0_{-10.7}^{+26.7}$ & $7.7-174.8$ \\
\hline 3HWC J1819-150 & 0.0 & $-2.90{ }_{-0.07}^{+0.08}{ }_{-0.03}^{+0.10}$ & $33.9 \pm 2.5_{-5.4}^{+10.3}$ & $2.2-62.5$ \\
\hline 3HWC J1825-134 & 0.0 & $-2.35 \pm 0.02_{-0.02}^{+0.11}$ & $125.4 \pm 3.4_{-18.3}^{+53.1}$ & $9.2-183.4$ \\
\hline 3HWC J1831-095 & 0.0 & $-2.61 \pm 0.06_{-0.03}^{+0.12}$ & $33.8_{-1.9}^{+1.8}{ }_{-5.3}^{+12.2}$ & $4.2-106.7$ \\
\hline 3HWC J1837-066 & 0.0 & $-2.73 \pm 0.02_{-0.03}^{+0.11}$ & $81.9 \pm 1.7_{-13.2}^{+25.2}$ & $2.2-57.3$ \\
\hline 3HWC J1843-034 & 0.0 & $-2.36 \pm 0.03{ }_{-0.02}^{+0.14}$ & $47.2 \pm 1.6{ }_{-7.2}^{+19.6}$ & $6.2-142.6$ \\
\hline 3HWC J1844-001 & 0.0 & $-2.76 \pm 0.12_{-0.03}^{+0.12}$ & $7.4 \pm 1.0_{-1.3}^{+2.4}$ & $1.9-51.2$ \\
\hline 3HWC J1847-017 & 0.0 & $-2.87 \pm 0.04_{-0.04}^{+0.10}$ & $27.7 \pm 1.3_{-5.1}^{+8.4}$ & $1.4-33.1$ \\
\hline $3 \mathrm{HWC}$ J1849+001 & 0.0 & $-2.17 \pm 0.04_{-0.01}^{+0.16}$ & $23.5 \pm 1.4_{-3.9}^{+11.6}$ & $9.9-195.3$ \\
\hline 3 HWC J1852+013 & 0.0 & $-2.79 \pm 0.08{ }_{-0.04}^{+0.13}$ & $14.5_{-1.1}^{+1.0}{ }_{-2.6}^{+4.4}$ & $1.7-40.2$ \\
\hline 3 HWC J1857+027 & 0.0 & $-2.83 \pm 0.03{ }_{-0.03}^{+0.10}$ & $35.9 \pm 1.2_{-6.5}^{+10.2}$ & $1.3-31.8$ \\
\hline $3 \mathrm{HWC}$ J1857+051 & 0.0 & $-3.03 \pm 0.12_{-0.04}^{+0.09}$ & $5.9 \pm 1.0_{-1.2}^{+1.8}$ & $0.7-15.5$ \\
\hline $3 \mathrm{HWC}$ J1907+085 & 0.0 & $-2.95 \pm 0.099_{-0.04}^{+0.09}$ & $8.4_{-1.0}^{+0.9}{ }_{-1.6}^{+2.5}$ & $0.8-19.7$ \\
\hline
\end{tabular}

Table 2 continued on next page 
Table 2 (continued)

\begin{tabular}{|c|c|c|c|c|}
\hline Name & $\begin{array}{c}\text { Radius } \\
\left.{ }^{\circ}\right]\end{array}$ & Index & $\begin{array}{c}F_{7} \\
{\left[10^{-15} \mathrm{TeV}^{-1} \mathrm{~cm}^{-2} \mathrm{~s}^{-1}\right]}\end{array}$ & $\begin{array}{c}\text { Energy Range } \\
{[\quad[\mathrm{TeV}]}\end{array}$ \\
\hline 3HWC J1908+063 & 0.0 & $-2.12 \pm 0.03_{-0.02}^{+0.18}$ & $44.7 \pm 1.4_{-7.1}^{+20.7}$ & $8.9-182.7$ \\
\hline 3HWC J1912+103 & 0.0 & $-2.85 \pm 0.05_{-0.03}^{+0.09}$ & $14.5 \pm 0.9_{-2.6}^{+4.4}$ & $1.1-27.1$ \\
\hline 3HWC J1913+048 & 0.0 & $-2.37 \pm 0.13{ }_{-0.03}^{+0.14}$ & $6.8_{-1.0}^{+0.9}{ }_{-1.1}^{+2.8}$ & $5.9-144.1$ \\
\hline 3HWC J1914+118 & 0.0 & $-2.9 \pm 0.1 \pm 0.1$ & $9.5_{-1.0}^{+0.9}{ }_{-1.9}^{+2.9}$ & $1.0-23.5$ \\
\hline 3HWC J1915+164 & 0.0 & $-2.60 \pm 0.13_{-0.01}^{+0.07}$ & $4.6 \pm 0.7_{-0.7}^{+1.4}$ & $2.5-69.5$ \\
\hline 3HWC J1918+159 & 0.0 & $-2.49_{-0.17}^{+0.15}{ }_{-0.02}^{+0.13}$ & $5.1 \pm 0.7_{-0.8}^{+1.8}$ & $3.6-101.9$ \\
\hline 3HWC J1920+147 & 0.0 & $-2.98 \pm 0.13_{-0.05}^{+0.11}$ & $6.0_{-1.0}^{+0.9}{ }_{-1.3}^{+2.0}$ & $0.7-16.8$ \\
\hline 3HWC J1922+140 & 0.0 & $-2.62 \pm 0.06_{-0.02}^{+0.09}$ & $13.0 \pm 0.8_{-2.0}^{+3.7}$ & $2.1-60.0$ \\
\hline 3HWC J1923+169 & 0.0 & $-3.07 \pm 0.10_{-0.03}^{+0.07}$ & $5.2 \pm 0.8_{-0.9}^{+1.4}$ & $0.5-11.1$ \\
\hline 3HWC J1928+178 & 0.0 & $-2.30 \pm 0.07_{-0.02}^{+0.17}$ & $13.6 \pm 0.9_{-2.1}^{+5.3}$ & $5.9-140.5$ \\
\hline 3HWC J1930+188 & 0.0 & $-2.76 \pm 0.07{ }_{-0.03}^{+0.09}$ & $10.2 \pm 0.8_{-1.7}^{+2.9}$ & $1.4-36.6$ \\
\hline 3HWC J1935+213 & 0.0 & $-2.73 \pm 0.16_{-0.03}^{+0.08}$ & $4.4 \pm 0.7_{-0.7}^{+1.3}$ & $1.6-43.0$ \\
\hline 3HWC J1936+223 & 0.0 & $-2.9 \pm 0.3_{-0.1}^{+0.2}$ & $4.1_{-1.2}^{+0.9}{ }_{-1.2}^{+1.5}$ & $0.9-20.9$ \\
\hline 3HWC J1937+193 & 0.0 & $-2.90 \pm 0.16_{-0.04}^{+0.09}$ & $4.2_{-0.8}^{+0.7}{ }_{-0.8}^{+1.2}$ & $0.9-22.2$ \\
\hline 3 HWC J1940+237 & 0.0 & $-3.14_{-0.11}^{+0.12}{ }_{-0.03}^{+0.07}$ & $4.0 \pm 0.8_{-0.8}^{+1.1}$ & $0.4-7.9$ \\
\hline 3HWC J1950+242 & 0.0 & $-2.49_{-0.19}^{+0.18}{ }_{-0.02}^{+0.15}$ & $4.3 \pm 0.7_{-0.7}^{+1.5}$ & $3.7-102.2$ \\
\hline 3HWC J1951+266 & 0.5 & $-2.36{ }_{-0.13}^{+0.12}{ }_{-0.02}^{+0.11}$ & $8.5_{-1.3}^{+1.2}{ }_{-1.3}^{+2.6}$ & $5.5-133.7$ \\
\hline 3HWC J1951+293 & 0.0 & $-2.47_{-0.10}^{+0.09}{ }_{-0.02}^{+0.10}$ & $7.9 \pm 0.8_{-1.2}^{+2.7}$ & $4.0-108.6$ \\
\hline 3HWC J1954+286 & 0.0 & $-2.42 \pm 0.12_{-0.02}^{+0.15}$ & $6.4 \pm 0.8_{-1.0}^{+2.4}$ & $4.8-119.7$ \\
\hline 3 HWC J1957+291 & 0.0 & $-2.54_{-0.14}^{+0.13}{ }_{-0.02}^{+0.11}$ & $6.2 \pm 0.7_{-1.0}^{+2.1}$ & $3.3-93.9$ \\
\hline 3 HWC J2005+311 & 0.0 & $-2.58 \pm 0.13_{-0.02}^{+0.11}$ & $5.6 \pm 0.7_{-0.9}^{+1.8}$ & $3.0-83.6$ \\
\hline 3HWC J2006+340 & 0.0 & $-2.56_{-0.13}^{+0.12}{ }_{-0.03}^{+0.15}$ & $8.5_{-0.9}^{+0.8}{ }_{-1.5}^{+3.1}$ & $3.3-83.5$ \\
\hline 3HWC J2010+345 & 0.0 & $-2.91 \pm 0.13_{-0.03}^{+0.10}$ & $5.4 \pm 0.9_{-0.9}^{+1.5}$ & $1.1-24.3$ \\
\hline 3 HWC J2019+367 & 0.0 & $-2.04 \pm 0.02_{-0.02}^{+0.16}$ & $34.7 \pm 1.3_{-5.7}^{+17.8}$ & $11.7-211.7$ \\
\hline 3HWC J2020+403 & 0.0 & $-3.11 \pm 0.07_{-0.04}^{+0.08}$ & $11.4 \pm 1.2_{-2.2}^{+3.4}$ & $0.7-14.8$ \\
\hline 3 HWC J2022+431 & 0.0 & $-2.34 \pm 0.12_{-0.02}^{+0.10}$ & $6.0 \pm 1.1_{-0.9}^{+2.2}$ & $8.3-181.9$ \\
\hline 3HWC J2023+324 & 1.0 & $-2.70_{-0.12}^{+0.13}{ }_{-0.03}^{+0.06}$ & $13.8_{-2.0}^{+1.9}{ }_{-2.1}^{+3.2}$ & $2.0-52.9$ \\
\hline 3 HWC J2031+415 & 0.0 & $-2.36 \pm 0.04_{-0.02}^{+0.14}$ & $30.7_{-1.4}^{+1.3}{ }_{-4.9}^{+13.1}$ & $6.3-147.8$ \\
\hline 3HWC J2043+443 & 0.5 & $-2.33_{-0.15}^{+0.14}{ }_{-0.02}^{+0.11}$ & $9.7_{-2.2}^{+2.1}{ }_{-1.5}^{+3.9}$ & $8.5-185.7$ \\
\hline $3 \mathrm{HWC}$ J2227+610 & 0.0 & $-2.43 \pm 0.10_{-0.02}^{+0.10}$ & $30.8_{-5.8}^{+5.9}{ }_{-4.1}^{+13.1}$ & $14.3-292.7$ \\
\hline
\end{tabular}




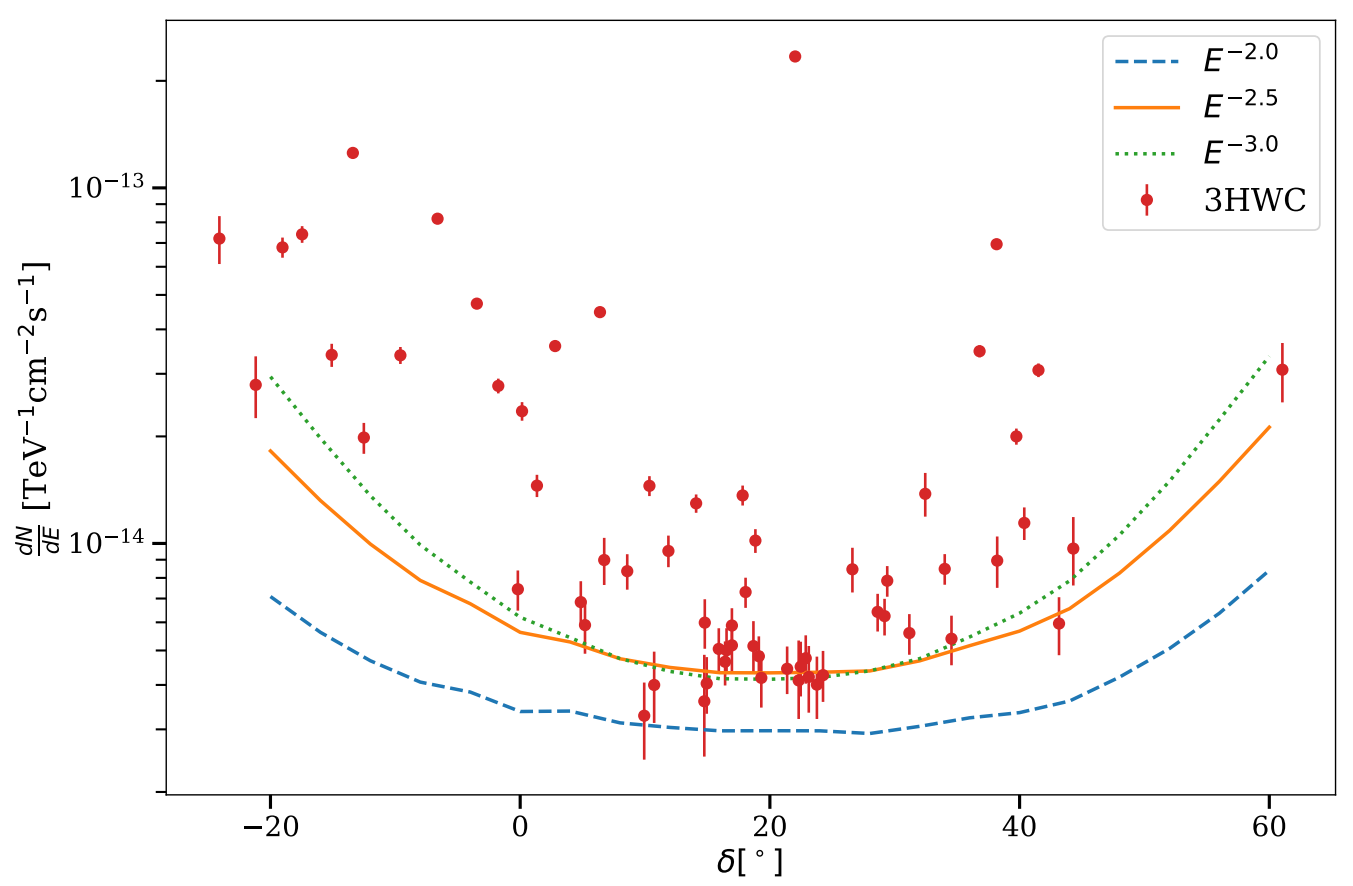

Figure 2. 3HWC sensitivity for the point source search as a function of declination. The flux sensitivity is shown at a pivot energy of $7 \mathrm{TeV}$ for three spectral hypotheses: $E^{-2.0}, E^{-2.5}$ and $E^{-3.0}$. The sensitivity does not depend on the Right Ascension. Also shown is the best-fit flux normalization at $7 \mathrm{TeV}$ for all sources in the $3 \mathrm{HWC}$ catalog.
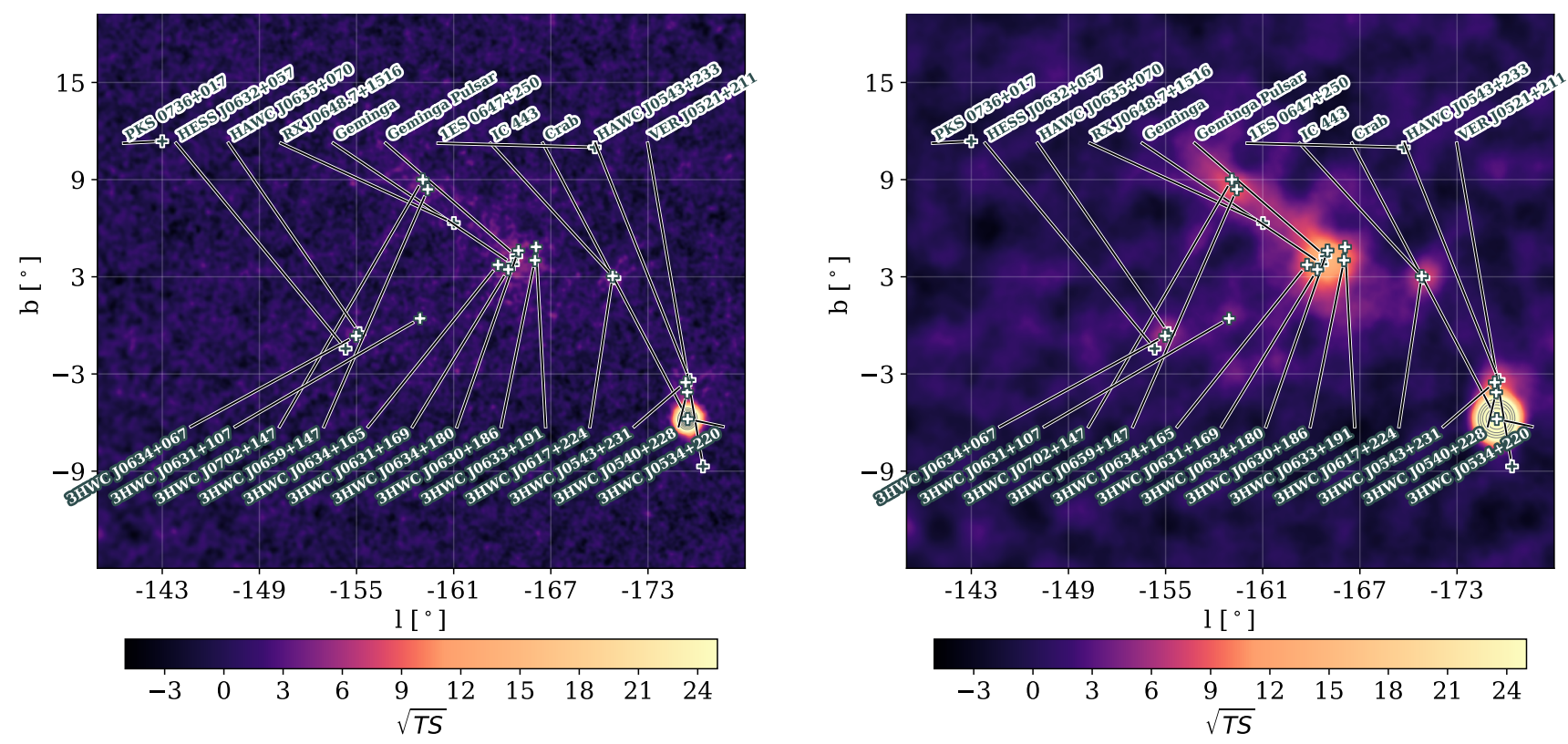

Figure 3. Significance maps of the Galactic anti-center region for $-176^{\circ} \leq l \leq-142^{\circ}$, showing the Crab Nebula and the Geminga halo among other sources. Left: Point-source hypothesis. Right: $1^{\circ}$ extended-source hypothesis. The grey lines show significance contours starting at $\sqrt{T S}=40$, increasing in steps of $\Delta \sqrt{T S}=20$. Top labels indicate positions of known TeV sources (from TeVCat), bottom labels indicate positions of $3 \mathrm{HWC}$ sources. 


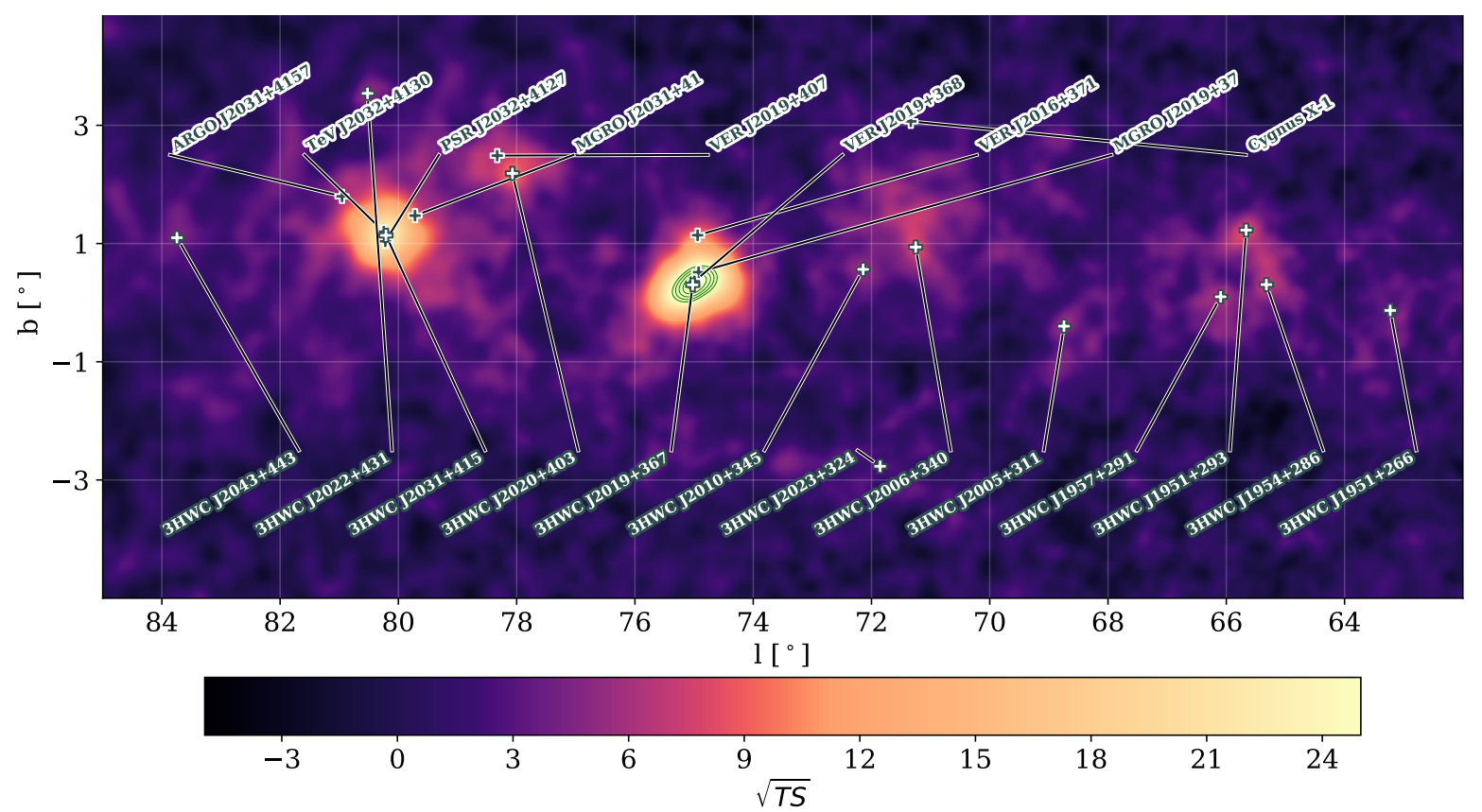

Figure 4. Significance map of part of the Galactic plane for $62^{\circ} \leq l \leq 85^{\circ}$; point-source hypothesis. The green lines show significance contours starting at $\sqrt{T S}=26$, increasing in steps of $\Delta \sqrt{T S}=2$. Top labels indicate positions of known TeV sources (from TeVCat), bottom labels indicate positions of $3 \mathrm{HWC}$ sources.

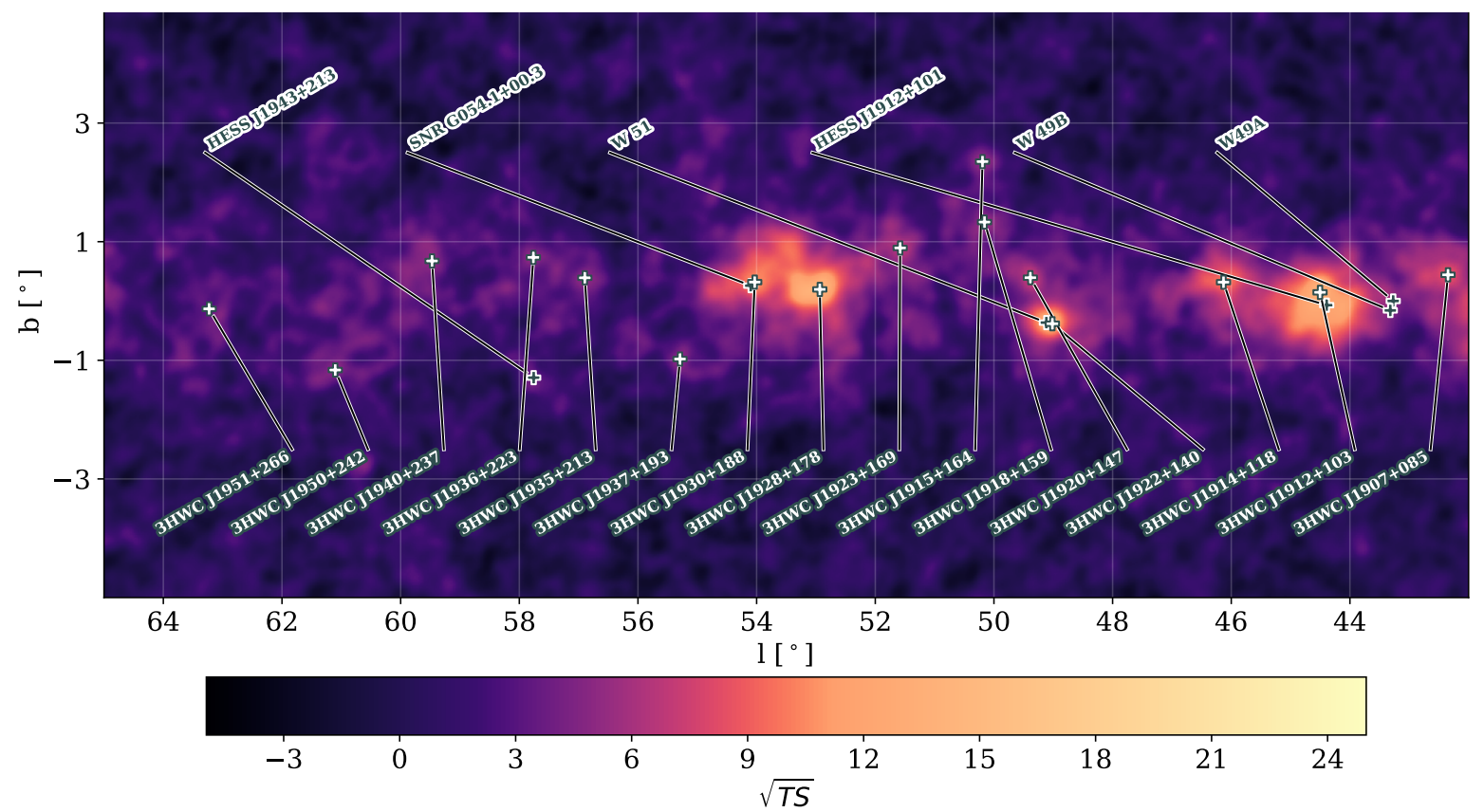

Figure 5. Significance map of part of the Galactic plane for $42^{\circ} \leq l \leq 65^{\circ}$; point-source hypothesis. Top labels indicate positions of known TeV sources (from TeVCat), bottom labels indicate positions of $3 \mathrm{HWC}$ sources. 


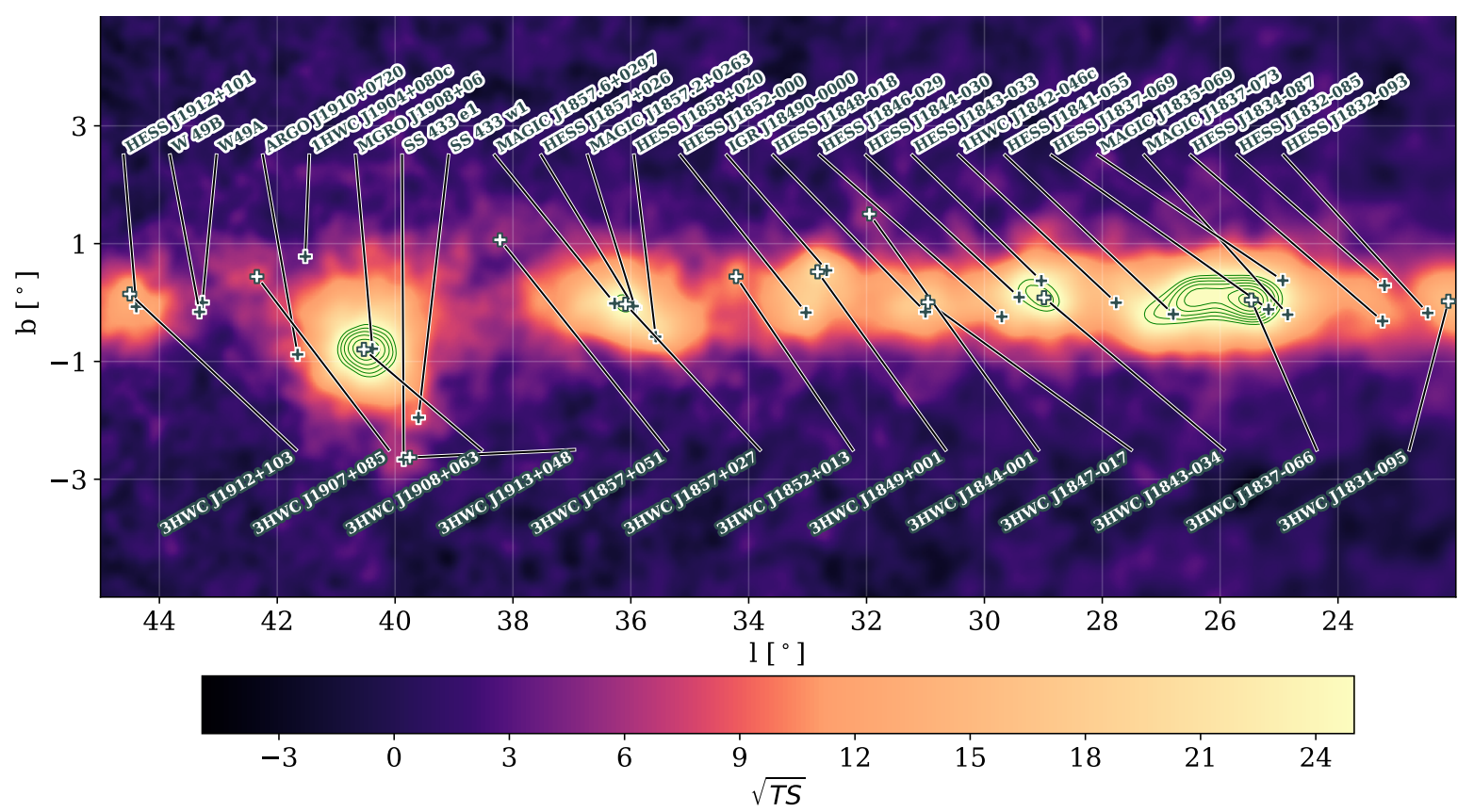

Figure 6. Significance map of part of the Galactic plane for $22^{\circ} \leq l \leq 45^{\circ}$; point-source hypothesis. The green lines show significance contours starting at $\sqrt{T S}=26$, increasing in steps of $\Delta \sqrt{T S}=2$. Top labels indicate positions of known TeV sources (from TeVCat), bottom labels indicate positions of $3 \mathrm{HWC}$ sources.

\subsection{Comparison with the $2 H W C$ catalog}

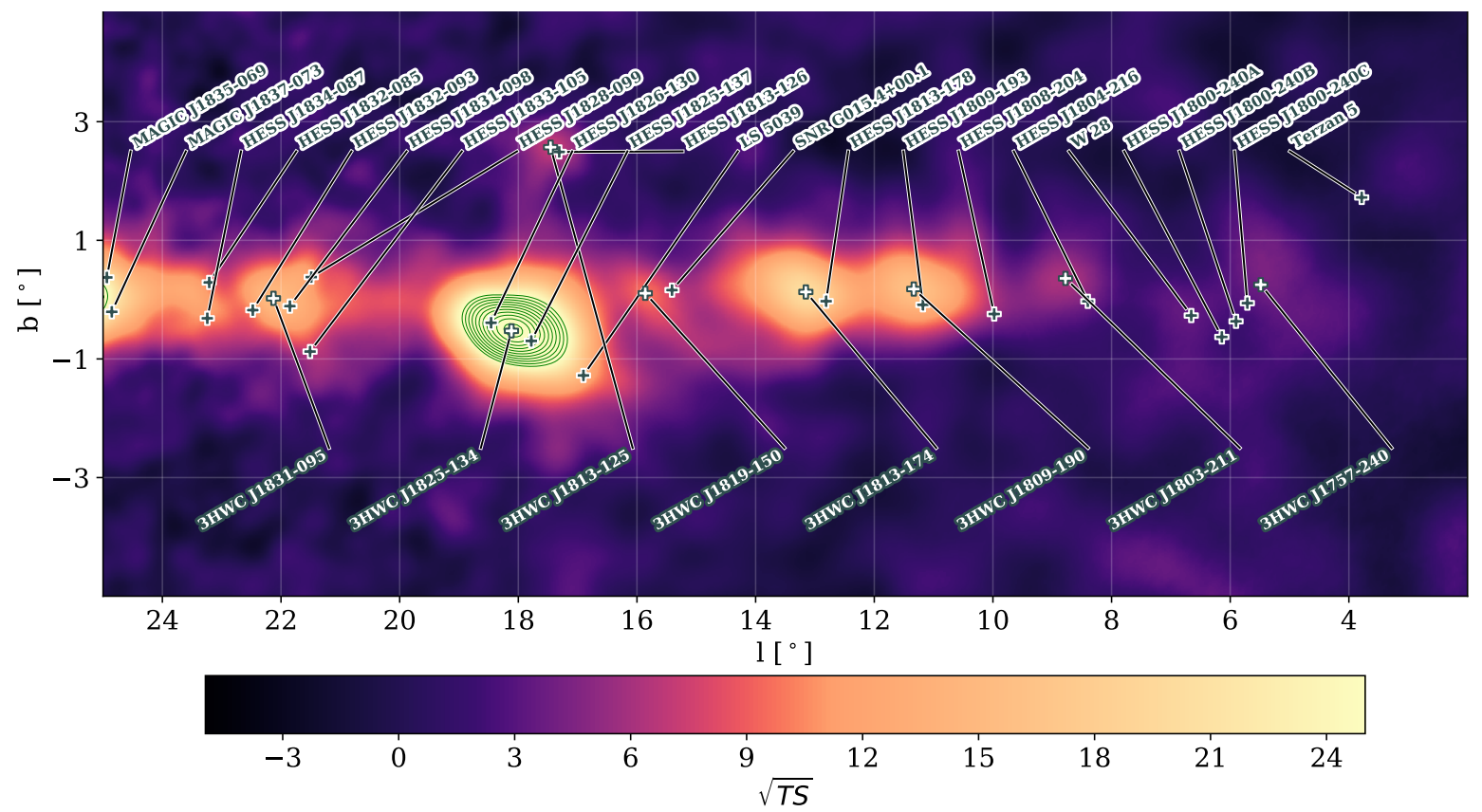

Figure 7. Significance map of the inner Galactic plane for $2^{\circ} \leq l \leq 25^{\circ}$; point-source hypothesis. The green lines show significance contours starting at $\sqrt{T S}=26$, increasing in steps of $\Delta \sqrt{T S}=2$. Top labels indicate positions of known TeV sources (from TeVCat), bottom labels indicate positions of $3 \mathrm{HWC}$ sources. 
Thirty-three of the 40 the sources detected in the $2 \mathrm{HWC}$ catalog have a $3 \mathrm{HWC}$ counterpart within $1^{\circ}$. Most of HAWC's sources are supernova remnants, pulsar wind nebula, or pulsar halos, and are expected to have constant emission, with the exception of the two Markarians, which are known to be variable at $\mathrm{TeV}$ energies on time scales of hours to weeks (e.g. Abeysekara et al. 2017d). For these sources, the TS increased by a factor of 2.3, on average. This is slightly less than the expected improvement due to the increase in livetime (factor of 3 ). The apparent deficit is explained by the new instrument response functions and the change in spectral index that was used in the source searches, verified by re-running the catalog search with the new settings on the $2 \mathrm{HWC}$ dataset.

There are seven $2 \mathrm{HWC}$ sources without a $3 \mathrm{HWC}$ counterpart within $1^{\circ}$. Two of these sources $(\mathbf{2 H W C ~} \mathbf{J 1 9 0 2 + 0 4 8}$ and $\mathbf{2 H W C ~ J 2 0 2 4 + 4 1 7}$ ) still show significant emission $(T S>25)$ in the new data set, but do not pass the TS dip test. 2HWC J1902+048 is now considered part of the 3HWC J1908+063 complex, and 2HWC J2024+417 is part of the 3HWC J2031+415 complex.

The remaining five sources do not pass the $T S>25$ criterion with the new data set. Out of these, $\mathbf{2 H W C}$ $\mathbf{J 1 9 2 1 + 1 3 1}$ lies in the Galactic plane near the W51 supernova remnant. In the 3HWC data set (point-source search), 2HWC J1921+131 has a TS of 21.7, which is below the threshold for significant detection. It is possible that the $2 \mathrm{HWC}$ detection was due to an upward fluctuation of a combination of diffuse emission and emission from the nearby W51 complex. The other four sources (2HWC J0819+157, 2HWC J1040+308, 2HWC J1309-054, and 2HWC $\mathbf{J 1 8 2 9 + 0 7 0}$ ) were located off the Galactic plane and had no known counterparts. Due to the low detection significance of these sources, searches for variability of these sources on time-scales of several months have not yielded conclusive results. (We note that these searches were mainly focused on a change in detection significance scaled over time. A more detailed analysis of the light-curves of these sources will follow in future HAWC publications.) It is unclear whether the previous detections were false positives due to random background fluctuations or upward fluctuations of real, but weak, sources, or indicative of flaring activity/temporal variability. We estimate the expected number of false positives, i.e. random background fluctuations that pass the detection threshold, to be 0.75 for the $3 \mathrm{HWC}$ catalog (see Section 4), compared to 0.4 for $2 \mathrm{HWC}$.

Four of the seven 2HWC sources without a 3 HWC equivalent $(\mathbf{2 H W C} \mathbf{J 0 8 1 9 + 1 5 7 ,} \mathbf{2 H W C} \mathbf{J 1 0 4 0 + 3 0 8}$, 2HWC J1829+070, and 2HWC J1902+048) are also not detected anymore when applying the new search methods/settings to the $2 \mathrm{HWC}$ dataset.

\subsection{New 3HWC Sources Potentially Associated with Known TeV Sources}

Eight of the $3 \mathrm{HWC}$ sources have no counterpart (within $1^{\circ}$ ) in $2 \mathrm{HWC}$, but are potentially associated with known $\mathrm{TeV}$ emitters. These sources are listed below. We define positional coincidence within $1^{\circ}$ as the criterion for two sources to be considered as candidates for association.

3HWC J0540+228 $(T S=28.8)$ and 3HWC J0543+231 $(T S=34.2)$ are part of the extended source that had been previously announced as HAWC J0543+233 (Riviere et al. 2017) - a potential TeV halo around the pulsar PSR B0540+23.

3HWC J0617+224 $(T S=32.3)$ is potentially associated with the shell-type supernova remnant (SNR) IC 443 (SNR G189.1+03.0), which has been detected at TeV energies by MAGIC (Albert et al. 2007) and VERITAS (Acciari et al. 2009a). HAWC previously announced the detection of gamma-ray emission from IC 443 without naming the source (Fleischhack 2020).

3HWC J0634+067 (TS $=36.2$ ) had been previously announced as HAWC J0635+070 (Brisbois et al. 2018) a potential $\mathrm{TeV}$ halo around the pulsar PSR J0633+0632.

3HWC $\mathbf{~} 2227+610(T S=52.5)$ is within $1^{\circ}$ of the known TeV gamma-ray sources VER J2227+608 (Acciari et al. 2009b) and MGRO J2228+61 (Abdo et al. 2009a,b). The most likely source of the emission is the shell-type supernova remnant SNR G106.3+2.7. 3HWC J2227+610 was recently announced as HAWC J2227+610 in a dedicated publication (Albert et al. 2020).

3HWC J1913+048 $(T S=44.7)$ is within $1^{\circ}$ of the eastern lobe of the micro-quasar SS 433. Detection of TeV gamma-ray emission from this source (as well as the western lobe of SS 433) had previously been announced by HAWC (Abeysekara et al. 2018).

3HWC J1757-240 (TS $=28.6)$ was found in the $1^{\circ}$ extended source search. It overlaps with the W28 region, which contains at least four known TeV sources (HESS J1801-233, and HESS J1800-240A/B/C) (Aharonian et al. 2008). The emission seen by H.E.S.S. has been attributed to an SNR interacting with nearby molecular clouds. 
Table 3. New HAWC sources with no TeV counterpart. For each source we list the following information in the various columns: Galactic longitude; Galactic latitude; the nearest GeV source in 4FGL (Abdollahi et al. 2020) and its separation from the 3HWC source; the source class as listed in 4FGL where available (bcu: active galaxy of uncertain type, PSR: pulsar, identified by pulsations, unk: unknown); the nearest pulsar and corresponding separation from the ATNF pulsar catalog (Manchester et al. 2005); and the nearest SNR, separation distance, and type from the SNRCat (Ferrand \& Safi-Harb 2012).

\begin{tabular}{|c|c|c|c|c|c|c|c|}
\hline HAWC & $l\left[^{\circ}\right]$ & $b\left[^{\circ}\right]$ & $4 \mathrm{FGL}\left({ }^{\circ}\right)$ & Class & $\operatorname{ATNF}\left({ }^{\circ}\right)$ & SNRCat $\left({ }^{\circ}\right)$ & SNR Type \\
\hline 3 HWC J0621+382 & 175.44 & 10.97 & 4FGL J0620.3+3804 (0.22) & bcu & J0622+3749 (0.42) & $\ldots$ & $\ldots$ \\
\hline 3 HWC J0630+186 & 193.98 & 4.02 & $\ldots$ & $\ldots$ & J0630+19 (0.94) & $\ldots$ & \\
\hline $3 \mathrm{HWC}$ J0631+107 & 201.08 & 0.43 & 4FGL J0631.5+1036 (0.15) & PSR & $\mathrm{J} 0631+1036(0.14)$ & $\ldots$ & $\ldots$ \\
\hline 3HWC J0633+191 & 193.92 & 4.85 & $\ldots$ & $\ldots$ & $\ldots$ & $\ldots$ & $\ldots$ \\
\hline 3HWC J1739+099 & 33.89 & 20.34 & 4FGL J1740.5+1005 (0.22) & PSR & $\mathrm{J} 1740+1000(0.13)$ & G034.0+20.3 (0.13) & filled-centre \\
\hline 3HWC J1743+149 & 39.13 & 21.68 & $\ldots$ & $\cdots$ & $\ldots$ & $\ldots$ & \\
\hline 3HWC J1844-001 & 31.95 & 1.50 & 4FGL J1848.2-0016 (0.99) & $\ldots$ & $\mathrm{J} 1843-0000(0.27)$ & $\cdots$ & $\cdots$ \\
\hline $3 \mathrm{HWC} \mathrm{J} 1857+051$ & 38.22 & 1.06 & 4FGL J1855.2+0456 (0.56) & $\ldots$ & $\mathrm{J} 1857+0526(0.24)$ & $\cdots$ & $\cdots$ \\
\hline 3 HWC J1915+164 & 50.19 & 2.35 & 4FGL J1912.0+1612 (0.74) & bcu & B1913+16 (0.32) & $\cdots$ & $\cdots$ \\
\hline 3HWC J1918+159 & 50.16 & 1.33 & $\ldots$ & $\ldots$ & $\mathrm{J} 1918+1541(0.26)$ & $\cdots$ & $\cdots$ \\
\hline 3HWC J1923+169 & 51.58 & 0.89 & 4FGL J1925.1+1707 (0.50) & unk & B1921+17 (0.14) & $\ldots$ & $\cdots$ \\
\hline 3 HWC J1935+213 & 56.90 & 0.39 & 4FGL J1935.2+2029 (0.89) & PSR & $\mathrm{J} 1936+21(0.24)$ & G057.2+00.8 (0.59) & shell \\
\hline 3 HWC J1936+223 & 57.76 & 0.73 & 4FGL J1932.2+2221 (0.94) & PSR & $\mathrm{J} 1938+2213(0.44)$ & G057.2+00.8 (0.47) & shell \\
\hline 3HWC J1937+193 & 55.29 & -0.98 & 4FGL J1936.6+1921 (0.21) & $\ldots$ & $\mathrm{J} 1936+20(0.77)$ & $\ldots$ & $\ldots$ \\
\hline 3 HWC J1951+266 & 63.23 & -0.13 & 4FGL J1951.6+2621 (0.25) & $\ldots$ & $\mathrm{J} 1952+2630(0.24)$ & $\ldots$ & $\ldots$ \\
\hline $3 \mathrm{HWC}$ J2005+311 & 68.74 & -0.40 & 4FGL J2006.2+3102 (0.15) & PSR & $\mathrm{J} 2006+3102(0.15)$ & G068.6-01.2 (0.81) & unknown \\
\hline $3 \mathrm{HWC}$ J2010+345 & 72.14 & 0.56 & $\ldots$ & $\ldots$ & $\ldots$ & $\ldots$ & $\ldots$ \\
\hline $3 \mathrm{HWC}$ J2022+431 & 80.52 & 3.54 & $\cdots$ & $\ldots$ & $\cdots$ & $\cdots$ & $\cdots$ \\
\hline $3 \mathrm{HWC}$ J2023+324 & 71.85 & -2.77 & 4FGL J2024.0+3202 (0.43) & unk & $\ldots$ & $\ldots$ & $\ldots$ \\
\hline $3 \mathrm{HWC}$ J2043+443 & 83.74 & 1.10 & 4FGL J2047.5+4356 (0.79) & $\ldots$ & $\ldots$ & $\ldots$ & $\ldots$ \\
\hline
\end{tabular}

Due to HAWC's limited sensitivity and relatively poor angular resolution at these declinations, we are currently unable to resolve the individual H.E.S.S. sources.

3HWC J1803-211 (TS = 38.4) is located near the known, but unidentified, TeV source HESS J1804-216 (Aharonian et al. 2005, 2006).

\subsection{New TeV Sources}

For each source in $3 \mathrm{HWC}$, we scan several catalogs of known or potential gamma-ray sources for potential associations within $1^{\circ}$ including the TeVCat (Wakely \& Horan 2008), the fourth Fermi-LAT source catalog (4FGL) (Abdollahi et al. 2020), the ATNF Pulsar Catalog ${ }^{2}$ (v 1.62) (Manchester et al. 2005), and the Galactic supernova remnant catalog SNRCat $^{3}$ (Ferrand \& Safi-Harb 2012)..

We report 20 new sources that do not have a potential counterpart in the TeVCat (see Table 3). Fourteen of these new sources are within $1^{\circ}$ of a previously observed $\mathrm{GeV}$ source. Table 3 lists the $\mathrm{GeV}$ associations and their source classifications obtained from the fourth Fermi-LAT source catalog, 4FGL. Two new sources, 3HWC J0630+186 $(T S=38.9)$ and $\mathbf{3 H W C ~} \mathbf{J 1 9 1 8}+\mathbf{1 5 9}(T S=31.6)$, have no GeV counterpart in 4FGL. These two sources are, however, potentially associated with pulsars in the ATNF catalog. $\mathbf{3 H W C ~} \mathbf{J 0 6 3 0}+\mathbf{1 8 6}$ is within $0.95^{\circ}$ of the pulsar PSR J0630+19. 3HWC J1918+159 is potentially associated with PSR J1918+1541 with a separation distance of $0.26^{\circ}$. The age and spin-down luminosity of these objects are not available.

\subsubsection{Unassociated New TeV Sources}

\footnotetext{
2 https://www.atnf.csiro.au/research/pulsar/psrcat/

${ }^{3}$ http://www.physics.umanitoba.ca/snr/SNRcat
} 


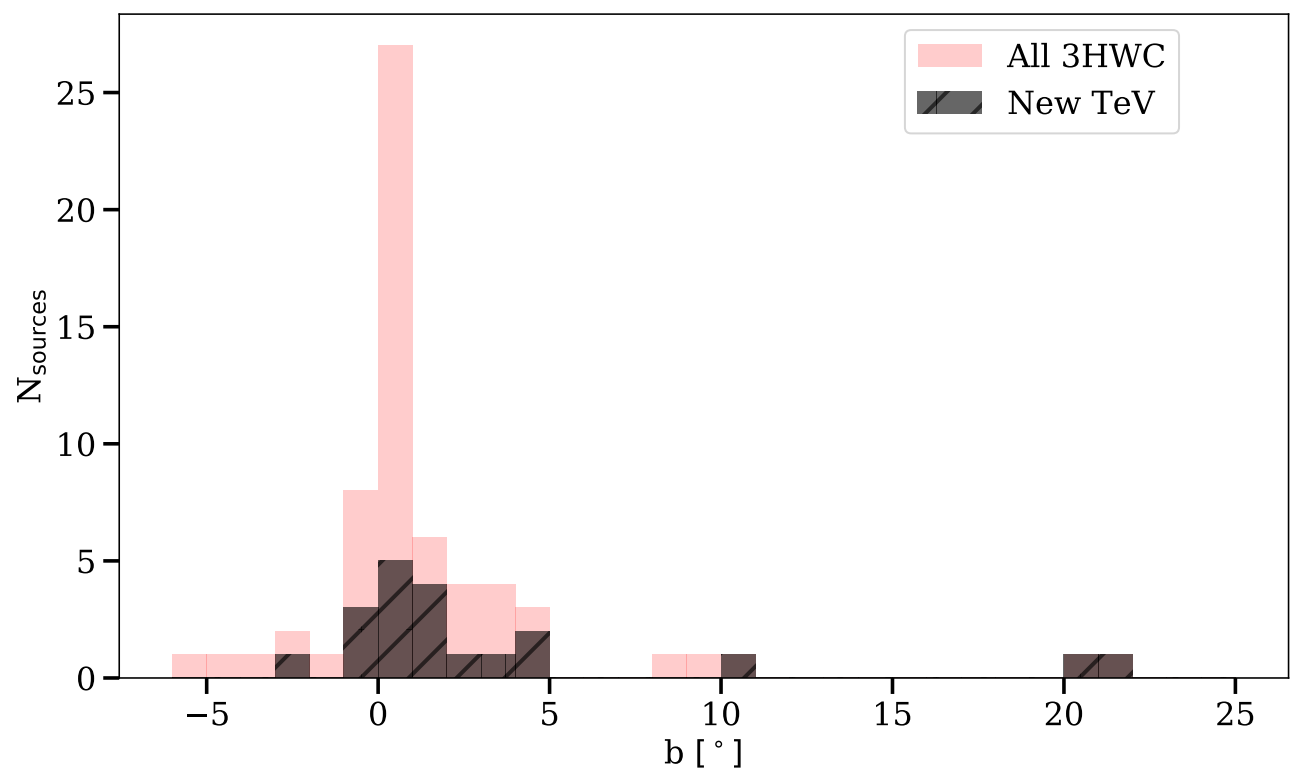

Figure 8. Distribution of HAWC sources (excluding the known blazars) with respect to galactic latitude for $-5^{\circ}<b<25^{\circ}$. The darker shaded histogram shows the new TeV sources in $3 \mathrm{HWC}$ that were not present in $2 \mathrm{HWC}$.

We observe four sources that do not have an apparent counterpart in any of the catalogs that we scanned for potential associations: 3HWC J0633+191 $(T S=37.5)$, 3HWC J2010+345 $(T S=27.6)$, 3HWC J2022+431 $(T S=29.0)$, and $\mathbf{3 H W C ~} \mathbf{J 1 7 4 3 + 1 4 9}(T S=25.9)$. We note that three of these new sources are not well isolated from known extended sources in the catalog. 3HWC J0633+191 is in a dense region of known pulsars including Geminga. 3HWC J2010+345 is near 3HWC J2004+343, which itself is a $1^{\circ}$ extended source. $3 \mathrm{HWC} \mathbf{J 2 0 2 2}+431$ is in the Cygnus-X region with a number of star-forming clusters nearby, most notably the Fermi-LAT cocoon (Hona et al. 2020). Without a detailed morphological study of their respective regions, we cannot definitively exclude the above new sources as appendages of existing sources. Such a study, however, is beyond the scope of this paper.

$\mathbf{3 H W C ~} \mathbf{J 1 7 4 3 + 1 4 9}$ (TS $=25.9$ ) is the only new unassociated source that is not in spatial proximity of a region of known TeV sources. It is also notably distant from the Galactic plane with a Galactic latitude of $b=21.7^{\circ}$. The nearest potential GeV gamma-ray counterpart is 4FGL J1741.4+1354, associated to the pulsar PSR J1741+1351, at a distance of $1.3^{\circ}$ from $3 \mathrm{HWC} \mathrm{J} 1743+149$ (outside of our nominal search radius for counterparts).

\subsubsection{Pulsars and TeV Halo Candidates in the $3 H W C$}

A significant fraction of $3 \mathrm{HWC}$ sources are candidates for association with pulsars in the ATNF catalog (c.f. Table 3). Figure 10 shows all the $3 \mathrm{HWC}$ sources potentially associated with pulsars in the galaxy for which the distance information is available.

After the discovery of extended gamma-ray emission around the Geminga and Monogem pulsars by HAWC (Abeysekara et al. 2017a), and the discovery of several extended TeV PWNe by H.E.S.S. (Abdalla et al. 2018), it has been suggested that extended pulsar "Halos" are a common feature or such objects and that several unassociated/not firmly associated HAWC sources may be dominated by such Halo emission (see e.g. Linden et al. 2017; Linden \& Buckman 2018; Giacinti et al. 2020; Sudoh et al. 2019; Fleischhack et al. 2019; Di Mauro et al. 2019; Manconi et al. 2020; Di Mauro et al. 2020). The observed gamma-ray emission is thought to be due to inverse Compton up-scattering of cosmic microwave background photons by relativistic electrons and positrons diffusing freely in the vicinity around the pulsar. TeV halos are thought to form around older pulsars (at least several tens of thousands of years old) that have either left their SNR shell or whose SNR shell has already dissipated. They are thus distinct from (classical) PWNe, where the electron-positron plasma is confined by the ambient medium.

We produce an updated list of pulsars that are likely candidates to have a TeV Halo detectable with HAWC, following similar criteria as Linden et al. (2017). We select pulsars from the ATNF with ages between $100 \mathrm{kyr}$ and 


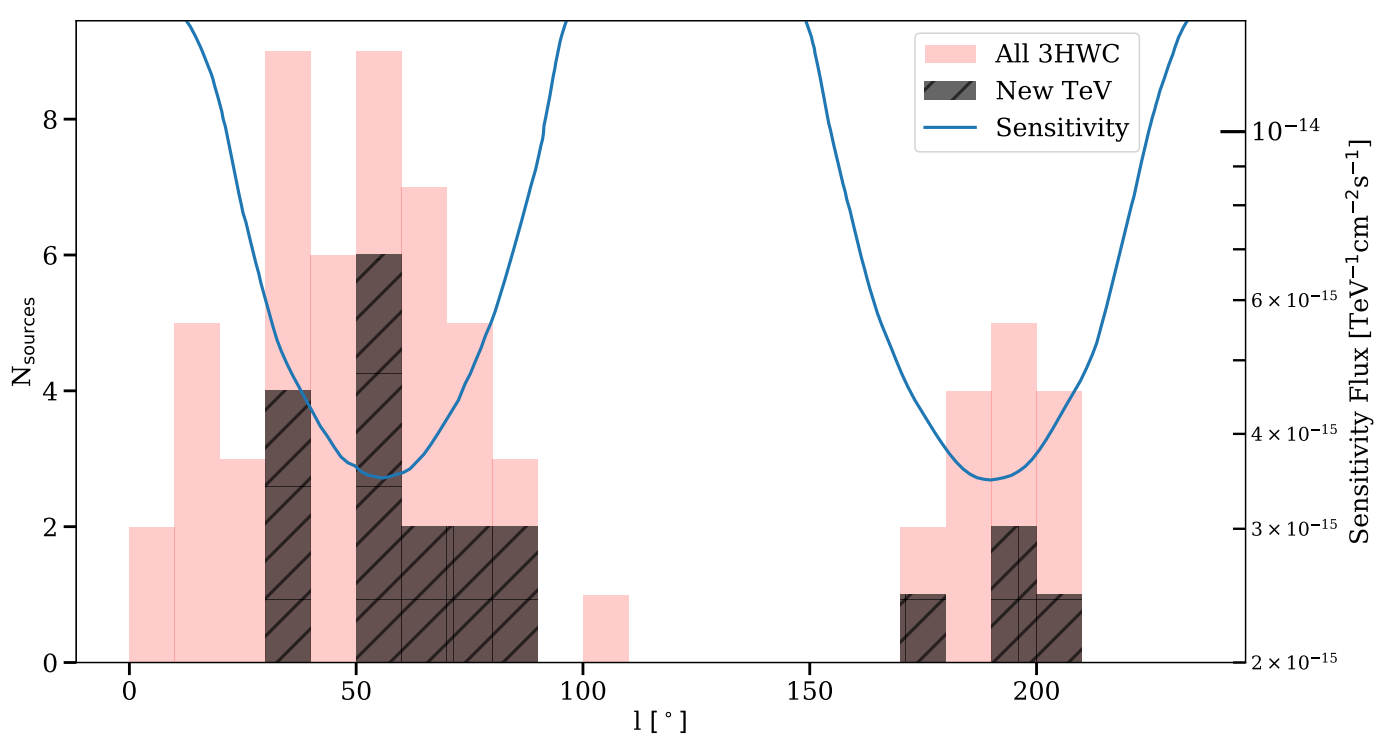

Figure 9. Distribution of HAWC sources as a function of galactic longitude. The darker shaded histogram shows the new $\mathrm{TeV}$ sources in $3 \mathrm{HWC}$ that were not present in $2 \mathrm{HWC}$. The blue solid line shows the sensitivity at $b=0^{\circ}$. Due to its location, HAWC is most sensitive towards the Galactic anticenter region, $l \approx-180^{\circ}$ and, to the inner Galaxy at $l \approx+50^{\circ}$. Most known $\mathrm{TeV}$ gamma-ray sources are located in the inner Galaxy.

Table 4. HAWC Sources with the corresponding TeV halo candidate pulsars within $1^{\circ}$. The age of the pulsar in kyr and the spin-down luminosity, $\dot{E}$, in $\mathrm{erg} \mathrm{s}^{-1}$ are also given. The Separation column indicates the angular distance between the HAWC source and the ATNF pulsar (Manchester et al. 2005). The TeVCat column lists the previously detected TeV counterpart of each source.

\begin{tabular}{|c|c|c|c|c|c|c|c|c|}
\hline HAWC & $l\left[^{\circ}\right]$ & $b\left[^{\circ}\right]$ & Pulsar & Age $[\mathrm{kyr}]$ & $\dot{E}\left[\operatorname{erg~s}^{-1}\right]$ & Distance [kpc] & Separation $\left[^{\circ}\right]$ & TeVCat \\
\hline 3 HWC J0540+228 & 184.58 & -4.13 & $\mathrm{~B} 0540+23$ & 253.0 & $4.09 \mathrm{e}+34$ & 1.56 & 0.83 & HAWC J0543+233 \\
\hline 3 HWC J0543+231 & 184.67 & -3.52 & B0540+23 & 253.0 & $4.09 \mathrm{e}+34$ & 1.56 & 0.36 & HAWC J0543+233 \\
\hline 3HWC J0631+169 & 195.63 & 3.45 & J0633+1746 & 342.0 & $3.25 \mathrm{e}+34$ & 0.19 & 0.95 & Geminga \\
\hline 3 HWC J0634+180 & 195.00 & 4.62 & J0633+1746 & 342.0 & $3.25 \mathrm{e}+34$ & 0.19 & 0.38 & Geminga Pulsar \\
\hline 3 HWC J0659+147 & 200.60 & 8.40 & B0656+14 & 111.0 & $3.8 \mathrm{e}+34$ & 0.29 & 0.51 & $2 \mathrm{HWC}$ J0700+143 \\
\hline 3 HWC J0702+147 & 200.91 & 9.01 & B0656+14 & 111.0 & $3.8 \mathrm{e}+34$ & 0.29 & 0.77 & 2HWC J0700+143 \\
\hline 3HWC J1739+099 & 33.89 & 20.34 & $\mathrm{~J} 1740+1000$ & 114.0 & $2.32 \mathrm{e}+35$ & 1.23 & 0.13 & $\ldots$ \\
\hline 3HWC J1831-095 & 22.13 & 0.02 & $\mathrm{~J} 1831-0952$ & 128.0 & $1.08 \mathrm{e}+36$ & 3.68 & 0.27 & HESS J1831-098 \\
\hline 3 HWC J1912+103 & 44.50 & 0.15 & $\mathrm{~J} 1913+1011$ & 169.0 & $2.87 \mathrm{e}+36$ & 4.61 & 0.31 & HESS J1912+101 \\
\hline 3HWC J1923+169 & 51.58 & 0.89 & $\mathrm{~J} 1925+1720$ & 115.0 & $9.54 \mathrm{e}+35$ & 5.06 & 0.67 & $\ldots$ \\
\hline 3 HWC J1928+178 & 52.93 & 0.20 & $\mathrm{~J} 1925+1720$ & 115.0 & $9.54 \mathrm{e}+35$ & 5.06 & 0.85 & $2 \mathrm{HWC}$ J1928+177 \\
\hline 3 HWC J2031+415 & 80.21 & 1.14 & $\mathrm{~J} 2032+4127$ & 201.0 & $1.52 \mathrm{e}+35$ & 1.33 & 0.11 & $\mathrm{TeV} \mathrm{J} 2032+4130$ \\
\hline
\end{tabular}

$400 \mathrm{kyr}$, declinations between $-25^{\circ}$ and $+64^{\circ}$, and an estimated spindown flux of at least $1 \%$ of that of the Geminga pulsar. We find sixteen such pulsars, out of which eight are coincident with at least one $3 \mathrm{HWC}$ source (within $1^{\circ}$ ). Table 4 lists the $3 \mathrm{HWC}$ sources that are coincident with these TeV Halo candidate pulsars. Some pulsars have more than one $3 \mathrm{HWC}$ source nearby. This is not unexpected as our source search sometimes finds multiple point sources associated with the same extended emission region. One of these pulsars, PSR J1740+1000, has not previously been detected at $\mathrm{TeV}$ energies. 


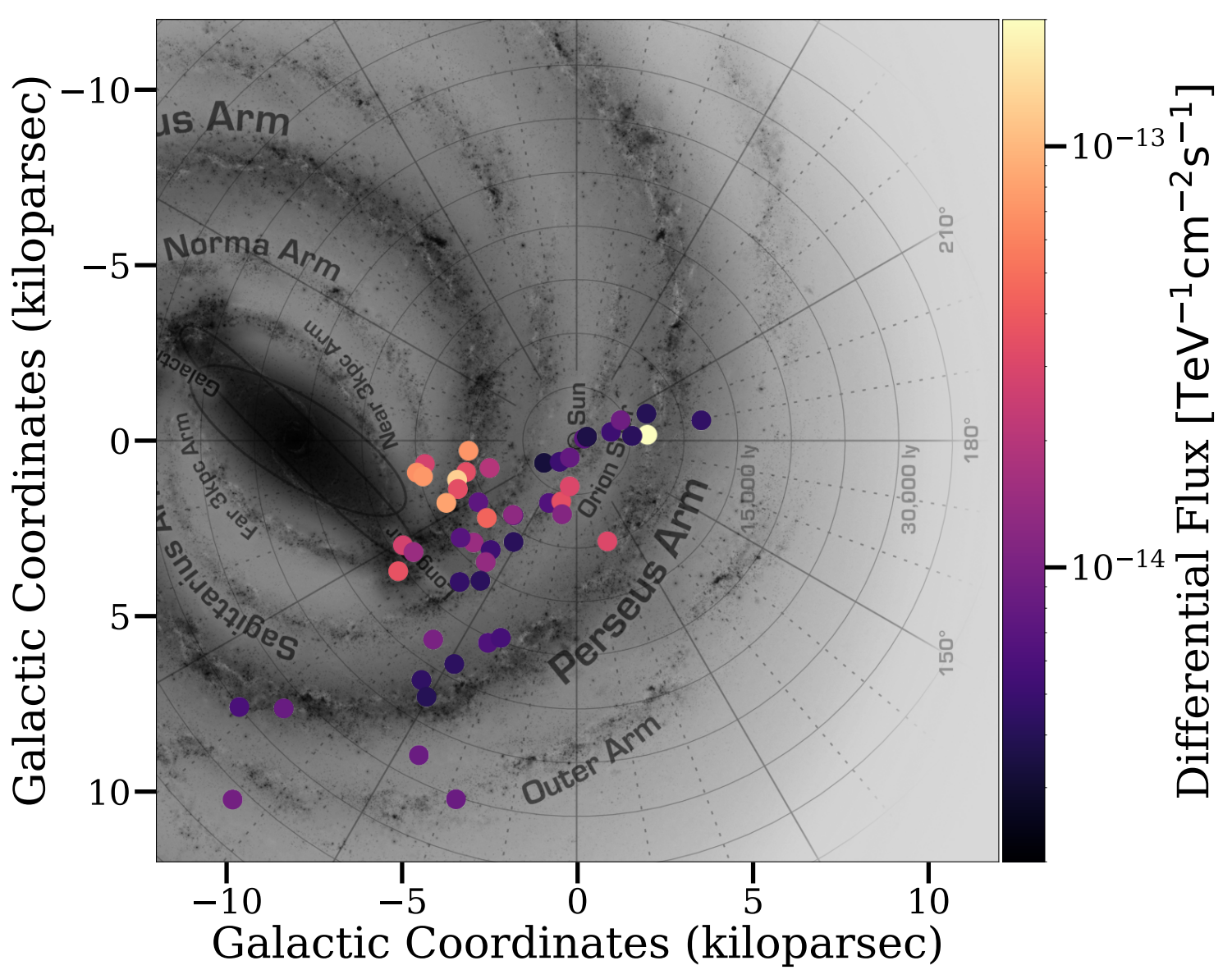

Figure 10. Face-on view of the galaxy showing positions of HAWC sources associated with (i.e., spatially coincident within $1^{\circ}$ of) pulsars for which distances are estimated. Spatial coincidence does not necessarily imply that the observed gamma-ray emission is (fully) powered by the pulsar in question. The color scale corresponds to the measured flux normalization from Table 2. The annotated Milky Way background is taken from NASA/JPL-Caltech/R.Hurt (2008).

\section{LIMITATIONS AND SYSTEMATIC UNCERTAINTIES}

\subsection{Background Fluctuations and Spurious Detections}

It is possible for mere fluctuations in the background and/or the Galactic diffuse emission to pass the selection criteria and produce a spurious source. In order to estimate the frequency of false positive sources, we create twenty simulated significance maps using the background counts from the original source search. For each map, we obtain the simulated number of signal events in each pixel by Poisson-fluctuating the number of background events in the corresponding pixel. We then run each of these randomized background maps through the full analysis pipeline, including point and extended searches. In the 20 total randomized background maps, we find 15 local maxima with a $T S>25$. Therefore, the estimated number of false positive sources is $15 / 20=0.75$. The fluctuations are distributed evenly across the sky and typically occur just above the threshold value of $T S=25$.

\subsection{Limitations of the Source Search}

As in the $2 \mathrm{HWC}$ catalog, we conduct blind source searches for four different fixed morphological assumptions (point sources, and $0.5^{\circ}, 1.0^{\circ}, 2.0^{\circ}$ extended sources). We then combine these results, with preference given to sources found in the point source search and the smaller radius searches to avoid double counting of sources.

This approach can lead to sources being misidentified or missed. First, some extended sources may be significant enough to be detected in the point source analysis. Poisson fluctuations of the signal could potentially lead to several hotspots being detected around the center of such an extended source. As HAWC collects more data, this issue is 
increasing in prevalence, as evidenced by the five point sources detected inside the Geminga halo. Second, it is also possible that multiple smaller sources located near each other are detected as one source in the extended source search if the individual sources are not strong enough to cross the detection threshold. This might be happening near the Galactic center where $\mathbf{3 H W C ~ J 1 7 5 7 - 2 4 0}$, found in the $1^{\circ}$ extended-source search, overlaps with several known TeV sources. Third, weaker sources may be missed if they are located near a stronger source, as they may not produce a well-defined peak in the significance map. In-depth studies (such as Abeysekara et al. (2017a, 2018)) are needed to properly resolve source-dense regions. Such studies include multi-source fits, fitting the extensions/shapes, locations, and spectra of several sources the same time. Additionally, measurements by other gamma-ray observatories as well as measurements at other wavelengths might help disentangle the morphology of complex regions. Further studies of selected regions of the Galactic plane are in preparation.

\subsection{Systematic Uncertainty on the Source Locations}

Earlier publications (Abeysekara et al. 2017b) quoted an $0.1^{\circ}$ systematic pointing uncertainty, which was estimated using simulations and verified through the observation of the Crab Nebula, Mrk421, and Mrk501. New studies of HAWC's pointing calibration as a function of source position suggest that the uncertainty could be larger than previously thought for sources that transit near the edge of HAWC's field of view. HAWC's absolute pointing uncertainty increases to $0.15^{\circ}$ for sources at $-10^{\circ}$ or $+50^{\circ}$ declination and could be as high as $0.3^{\circ}$ at declinations of $-20^{\circ}$ or $+60^{\circ}$. (There are no well-isolated point sources detected by HAWC that could be used to unambiguously verify the instrument's pointing at these declinations.)

In Figure 11, we compare the measured declinations of 3HWC sources to the locations of their likely TeV counterparts as measured by other experiments. For this comparison, we consider relatively well-localised 3HWC sources that have a $\mathrm{TeV}$ association within $1^{\circ}$ detected by a different experiment. We do not include sources in regions of extended

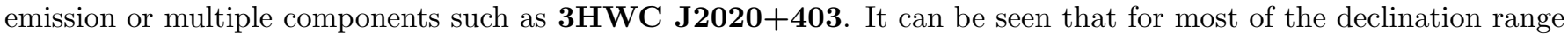
spanned by HAWC's sensitivity, the 3HWC positions agree with the literature values within statistical and systematic uncertainties. Below source declinations of about $-10^{\circ}$, HAWC measures systematically higher values than the IACTs, between an offset of $0.1^{\circ}$ and $0.4^{\circ}$.

The trend observed in Figure 11 could indicate a bias in HAWC's pointing at low declinations. However, all of HAWC's southern sources lie on the Galactic plane, in a region rich in sources and diffuse emission. HAWC's angular resolution is poor for low-declination sources compared to sources transiting overhead. Accordingly, Galactic diffuse emission or emission from nearby unresolved sources might affect the peak position detected by HAWC, especially for low-declination sources. IACTs tend to have better angular resolution and are thus affected less by large-scale emission or neighboring sources. The shift could also be an indication of an energy-dependent morphology of some sources Hona et al. (2020). Future in-depth studies of some of these sources as well as an improved understanding of HAWC's pointing are needed to resolve this apparent discrepancy.

\subsection{Systematic Uncertainty of the Spectral Fits}

In Table 2, we report the best-fit fluxes and spectral indices of the 3HWC sources. These fits assume a power-law spectrum; we do not test other spectral models including a curvature or cutoff term. The reported spectral index should be interpreted as an average or effective spectral index across HAWC's energy range. For the two known extragalactic sources, we also do not account for absorption by the extra-galactic background light. Additional studies are ongoing for sources that are detected with sufficient statistics to allow more sophisticated spectral models to be fit.

In Table 2, we report systematic uncertainties related to the modeling of the HAWC detector response individually for each source. More details about the sources of uncertainty considered here can be found in Abeysekara et al. (2019). In order to compute the uncertainties, we repeat spectral fits with certain properties of the detector model shifted up or down. We assign an additional uncertainty of $10 \%$ to the flux normalization to account for effects, such as variations in the atmosphere, that are not considered otherwise. We add the resulting positive shifts to the spectral fit parameters in quadrature to obtain the total upward systematic uncertainty, and add the negative shifts in quadrature for the total negative downward systematic uncertainty.

There are other systematic issues affecting the spectral fits. Some fluxes may be overestimated due to "leakage" from nearby (detected or unresolved) sources or from the Galactic diffuse emission. These may also affect the spectral indices. In cases where the apparent extent of a source is larger than HAWC's angular resolution $\left(0.1^{\circ}\right.$ at high energies), but the source is strong enough to be significantly detected already in the point-source search, we only 


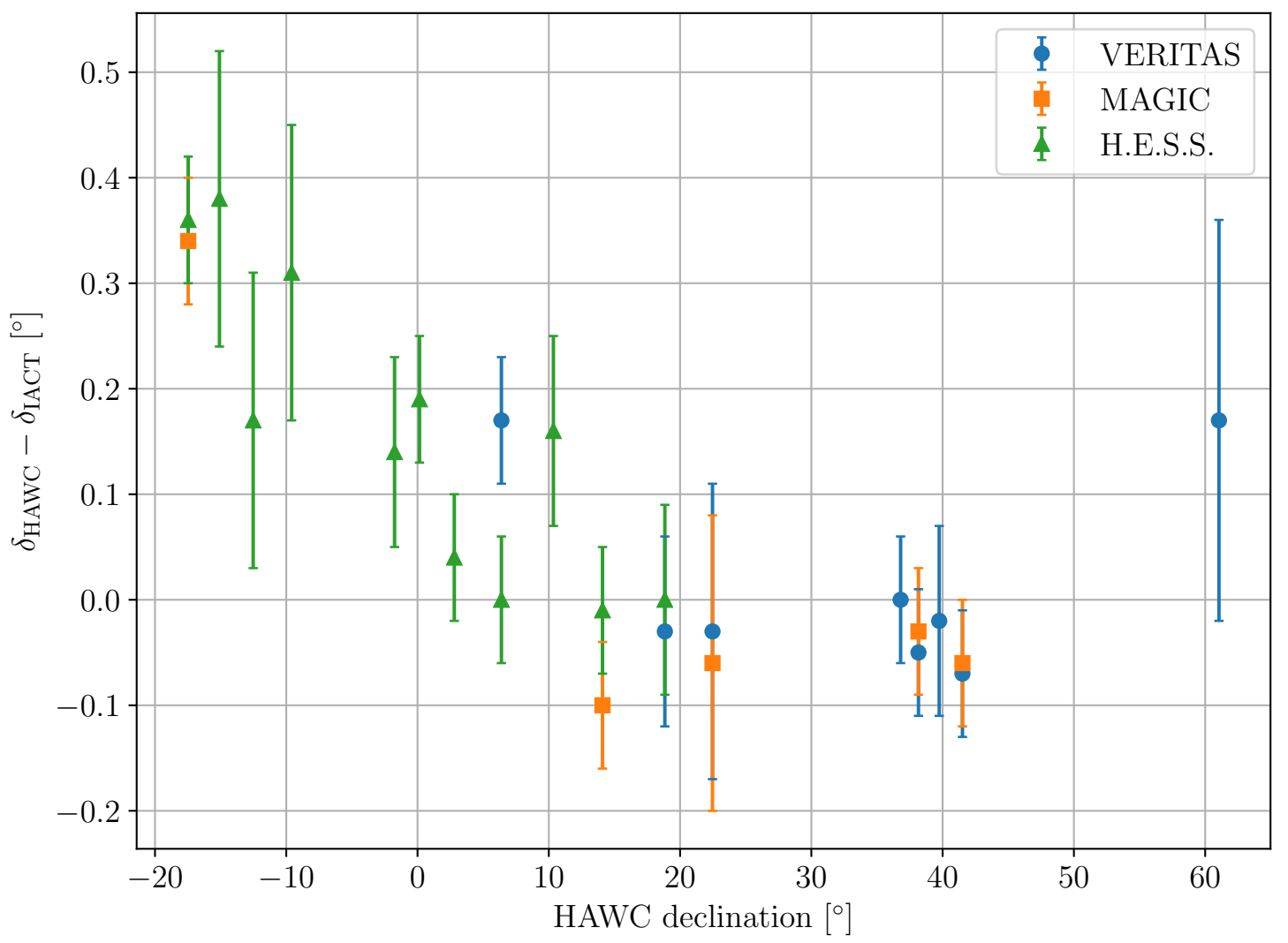

Figure 11. Measured declination of HAWC sources relative to their TeV counterpart measurements from IACT experiments, MAGIC, H.E.S.S. and VERITAS. HAWC measurements agree with the source locations measured by IACTs within uncertainties for most of its declination range. See text for discussion on source declinations below $-10^{\circ}$.

report the spectrum assuming a point-source hypothesis. This leads to the flux normalization being underestimated and the spectral index to be biased towards softer spectra (as the angular resolution improves for high energies and thus more of the high-energy emission is "lost"). Upcoming publications will provide better spectral fits for extended sources.

\section{CONCLUSION}

The HAWC observatory has been conducting the most sensitive, unbiased survey of the Northern sky at TeV energies for over five years. We have presented the third catalog of steady gamma-ray emitters detected by HAWC using 1523 days of data. The catalog consists of 65 sources, including two blazars. The most abundant source class among the potential counterpart of HAWC sources in the Galactic plane is pulsars (56).

The 3HWC catalog provides many targets for multi-wavelength and multi-messenger follow-up studies that are crucial to several open problems in high-energy astrophysics. Detailed morphological and spectral studies of several sources are being conducted and will be the subject of future publications. A dedicated survey to constrain the emission from various extra-galactic objects of interest is under preparation. Future gamma-ray observatories such as CTA (Acharya et al. 2018) and SWGO (Albert et al. 2019) will be able to extend both the sensitivity and energy range of this survey. 


\section{ACKNOWLEDGMENTS}

We acknowledge the support from: the US National Science Foundation (NSF); the US Department of Energy Office of High-Energy Physics; the Laboratory Directed Research and Development (LDRD) program of Los Alamos National Laboratory; Consejo Nacional de Ciencia y Tecnología (CONACyT), México, grants 271051, 232656, 260378, 179588, 254964, 258865, 243290, 132197, A1-S-46288, A1-S-22784, cátedras 873, 1563, 341, 323, Red HAWC, México; DGAPAUNAM grants IG101320, IN111315, IN111716-3, IN111419, IA102019, IN112218; VIEP-BUAP; PIFI 2012, 2013, PROFOCIE 2014, 2015; the University of Wisconsin Alumni Research Foundation; the Institute of Geophysics, Planetary Physics, and Signatures at Los Alamos National Laboratory; Polish Science Centre grant, DEC-2017/27/B/ST9/02272; Coordinación de la Investigación Científica de la Universidad Michoacana; Royal Society - Newton Advanced Fellowship 180385; Generalitat Valenciana, grant CIDEGENT/2018/034; Chulalongkorn University's CUniverse (CUAASC) grant. Thanks to Scott Delay, Luciano Díaz and Eduardo Murrieta for technical support.

\section{REFERENCES}

Abdalla, H., Abramowski, A., Aharonian, F., et al. 2018, Astron. Astrophys., 612, A2, doi: 10.1051/0004-6361/201629377

Abdo, A. A., Allen, B. T., Aune, T., et al. 2009a, The Astrophysical Journal, 700, L127, doi: 10.1088/0004-637x/700/2/1127

—. 2009b, The Astrophysical Journal, 703, L185, doi: 10.1088/0004-637x/703/2/1185

Abdollahi, S., Acero, F., \& Ackermann, M. o. 2020, Astrophys. J. Suppl., 247, 33, doi: 10.3847/1538-4365/ab6bcb

Abeysekara, A., Albert, A., Alfaro, R., et al. 2017a, Science, 358, 911, doi: 10.1126/science.aan4880

—. 2018, Nature, 562, 82, doi: 10.1038/s41586-018-0565-5

Abeysekara, A. U., Albert, A., Alfaro, R., et al. 2017b, The Astrophysical Journal, 843, 40, doi: $10.3847 / 1538-4357 /$ aa7556

Abeysekara, A. U., et al. 2017c, Astrophys. J., 843, 39, doi: 10.3847/1538-4357/aa7555

Abeysekara, A. U., Albert, A., Alfaro, R., et al. 2017d, The Astrophysical Journal, 841, 100, doi: 10.3847/1538-4357/aa729e

—. 2019, The Astrophysical Journal, 881, 134, doi: 10.3847/1538-4357/ab2f7d

Acciari, V. A., Aliu, E., Arlen, T., et al. 2009a, ApJL, 698, L133, doi: 10.1088/0004-637X/698/2/L133

—. 2009b, The Astrophysical Journal, 703, L6, doi: 10.1088/0004-637X/703/1/L6

Acharya, B., Agudo, I., Al Samarai, I., et al. 2018, Science with the Cherenkov Telescope Array (WSP), doi: 10.1142/10986

Aharonian, F., Akhperjanian, A. G., Aye, K. M., et al. 2005, Science, 307, 1938, doi: 10.1126/science.1108643

Aharonian, F., Akhperjanian, A. G., Bazer-Bachi, A. R., et al. 2006, ApJ, 636, 777, doi: 10.1086/498013
—. 2008, A\&A, 481, 401, doi: 10.1051/0004-6361:20077765

Ahnen, M. L., Ansoldi, S., Antonelli, L., et al. 2017, Astroparticle Physics, 94, 29, doi: 10.1016/j.astropartphys.2017.08.001

Albert, A., Alfaro, R., Ashkar, H., et al. 2019. https://arxiv.org/abs/1902.08429

Albert, A., Alfaro, R., Alvarez, C., et al. 2020, The Astrophysical Journal, 896, L29, doi: $10.3847 / 2041-8213 /$ ab96cc

Albert, J., Aliu, E., Anderhub, H., et al. 2007, ApJL, 664, L87, doi: 10.1086/520957

Amenomori, M., Bao, Y. W., Bi, X. J., et al. 2019, Phys. Rev. Lett., 123, 051101, doi: 10.1103/PhysRevLett.123.051101

Anderhub, H., Backes, M., A., B., et al. 2013, Journal of Instrumentation, 8, P06008, doi: 10.1088/1748-0221/8/06/P06008

Atkins, R., Benbow, W., Berley, D., et al. 2003, ApJ, 595, 803, doi: 10.1086/377498

Balzer, A., Füßling, M., Gajdus, M., et al. 2014, Astroparticle Physics, 54, 67, doi: 10.1016/j.astropartphys.2013.11.007

Bartoli, B., Bernardini, P., Bi, X., et al. 2013, Astrophys. J., 779, 27, doi: 10.1088/0004-637X/779/1/27

Brisbois, C., Riviere, C., Fleischhack, H., \& Smith, A. 2018, HAWC detection of TeV source HAWC J0635+070. http://www.astronomerstelegram.org/?read=12013

Di Mauro, M., Manconi, S., \& Donato, F. 2019, Phys. Rev. D, 100, 123015, doi: 10.1103/PhysRevD.100.123015

—. 2020, Phys. Rev. D, 101, 103035, doi: 10.1103/PhysRevD.101.103035

Ferrand, G., \& Safi-Harb, S. 2012, Advances in Space Research, 49, 1313, doi: 10.1016/j.asr.2012.02.004

Fleischhack, H. 2020, PoS, ICRC2019, 674. https://arxiv.org/abs/1907.08575 
Fleischhack, H., Albert, A., Alvarez, C., et al. 2019. https://arxiv.org/abs/1903.07647

Giacinti, G., Mitchell, A., López-Coto, R., et al. 2020, Astron. Astrophys., 636, A113, doi: 10.1051/0004-6361/201936505

Gorski, K. M., Hivon, E., Banday, A. J., et al. 2005, The Astrophysical Journal, 622, 759, doi: 10.1086/427976

Holder, J., Acciari, V., Aliu, E., et al. 2009, AIP Conf. Proc., 1085, 657, doi: 10.1063/1.3076760

Hona, B., Fleischhack, H., \& Huentemeyer, P. 2020, PoS, ICRC2019, 699, doi: 10.22323/1.358.0699

Linden, T., Auchettl, K., Bramante, J., et al. 2017, Phys. Rev. D, 96, 103016, doi: 10.1103/PhysRevD.96.103016

Linden, T., \& Buckman, B. J. 2018, Phys. Rev. Lett., 120, 121101, doi: 10.1103/PhysRevLett.120.121101

Manchester, R. N., Hobbs, G. B., Teoh, A., \& Hobbs, M. 2005, Astron. J., 129, 1993, doi: 10.1086/428488

Manconi, S., Di Mauro, M., \& Donato, F. 2020, Phys. Rev. D, 102, 023015, doi: 10.1103/PhysRevD.102.023015
Martinez-Castellanos, I. 2019, PhD thesis, University of Maryland. https://doi.org/10.13016/bcjz-xvre

NASA/JPL-Caltech/R.Hurt. 2008, Artist's impression of the Milky Way (updated - annotated). https://commons.wikimedia.org/wiki/File: 236084main_MilkyWay-full-annotated.jpg

Riviere, C., Fleischhack, H., \& Sandoval, A. 2017, HAWC detection of TeV emission near PSR B0540+23.

http://www.astronomerstelegram.org/?read=10941

Sudoh, T., Linden, T., \& Beacom, J. F. 2019, Phys. Rev. D, 100, 043016, doi: 10.1103/PhysRevD.100.043016

Wakely, S. P., \& Horan, D. 2008, in International Cosmic Ray Conference, Vol. 3, International Cosmic Ray Conference, 1341-1344

Wilks, S. S. 1938, Ann. Math. Statist., 9, 60, doi: 10.1214/aoms/1177732360

Younk, P. W., Lauer, R. J., Vianello, G., et al. 2015, in International Cosmic Ray Conference, Vol. 34, 34th International Cosmic Ray Conference (ICRC2015), 948. https://arxiv.org/abs/1508.07479

Zhen, C. 2014, Frascati Phys. Ser., 58, 331 